\title{
Type specimens of amphibians in the National Museum of Natural History, Leiden, The Netherlands
}

\author{
M. EULÀLIA GASSÓ MIRACLE, LARS W. VAN DEN HOEK OSTENDE \& JAN WILLEM ARNTZEN \\ M.E. Gassó Miracle: Department of Zoology, National Museum of Natural History Naturalis, Postbus 9517, 2300 RA Leiden, The \\ Netherlands. E-mail: gassomiracle@naturalis.nl \\ L.W. van den Hoek Ostende: Department of Geology, National Museum of Natural History Naturalis, Postbus 9517, 2300 RA Leiden, \\ The Netherlands. E-mail: hoek@naturalis.nl \\ J.W. Arntzen: Department of Zoology, National Museum of Natural History Naturalis, Postbus 9517, 2300 RA Leiden, The Nether- \\ lands.E-mail:arntzen@naturalis.nl
}

\begin{abstract}
The amphibian type specimens held in the National Museum of Natural History in Leiden are listed. A total of 775 type specimens representing 143 taxon names were encountered. The list provides the original name, the original publication date, pagination and illustrations, current name, type locality and notes on the type status.
\end{abstract}

Key words: Amphibia, Caudata, Anura, Gymnophiona, types, National Museum of Natural History, Leiden, Rijksmuseum van Natuurlijke Historie

\section{Introduction}

The National Museum of Natural History "Naturalis" in Leiden (until 1988 known as Rijksmuseum van Natuurlijke Historie, RMNH) was established in 1820 when three pre-existing collections were merged: the Cabinet du Roi of Louis Napoleon (or 's Lands Kabinet van Natuurlijke Historie), the private collection owned by C. J. Temminck and the collection of the University of Leiden, also known as the Academic Cabinet. Coenraad Jacob Temminck, ornithologist and founder of the museum, was then appointed director, and continued in that post until his death in 1858. The history of these early collections is described in The history of Natural History in Leiden (Van der Laan, 2001).

The collection of amphibians in the RMNH goes largely back to the $19^{\text {th }}$ century. The herpetological collection includes material from South East Asia and the Amazonian region, as well as several valuable collections from other localities. The oldest Asian material dates from the $1820 \mathrm{~s}$, and it was gathered by the members of the Natuurkundige Commissie, a group of naturalists sent to the Dutch East Indies to study and collect animals and plants for the museum. The first expedition began in July 1820. H. Kuhl, J. C. van Hasselt, both naturalists, together with J. Keultjes, artist, and G. van Raalten, taxidermist, arrived at Java and started the exploration from their basis in Buitenzorg (now Bogor). Unfortunately, they all died shortly after arrival. Kuhl and Keultjes died of tropical diseases after nine months and Van Hasselt in 1823. Replacement was sent in 1825: the zoologists H. Boie and H. Macklot, the taxidermist S. Müller and the artist P. Van Oort. Of this group, only Müller would return to Holland, after twelve years in East India. Boie died shortly after his arrival, in 1827, and so did Macklot, who died in 1832 after a fight with Chinese rebels. The Natuurkundige Commissie explored most of Java and part of Sumatra, Sulawesi and Papua. After Macklot's death, other zoologists, botanists and artists were sent to Java. Most of them felt victim of fatal disease and few of them 
returned. The Commission continued to explore the Dutch colonies until its dissolution in 1850. A concise history of the Natuurkundige Commissie can be found in Streseman's book about the development of Ornithology (1951).

After the dissolution of the Natuurkundige Commissie, the Leiden museum acquired zoological material from members of trade and exploratory voyages to the Dutch colonies, especially to the East Indies. The museum also received material from Western Europe, Africa and South America, collected by Dutch diplomats, army officials and private collectors. For example, the doctor H. A. Bernstein collected ethnological and zoological material in Halmahera and the surrounding islands, from 1860 to his death in 1865 , for the National Museum of Ethnology (Leiden) and the Museum of Natural History. The Swiss zoologist J. Büttikofer (who would later become the director of the Zoological Garden at Rotterdam) sent material from Liberia, collected between 1879 and 1887, and from central Borneo, collected in 1893-94 during the Nieuwenhuis-Expedition. Of special importance are the specimens sent from Japan by the German physicist Philipp F. von Siebold and his assistant, Heinrich Bürger. The Von Siebold collection of Japanese natural history specimens is the oldest in Europe and rich in type specimens. Director C. J. Temminck and curator Hermann Schlegel described several new species of vertebrates, including Japanese salamanders, in the well-known Fauna Japonica, that was published in Leiden between 1833 and 1850. More information on the Von Siebold collection can be found in Holthuis \& Sakai (1970).

The Leiden collection includes many amphibians that were described by the Swiss naturalist J. J. von Tschudi in his Classification der Batrachier, published in 1838. Tschudi collaborated with Schlegel in Leiden in 1837, and during his stay he examined and described several specimens of the Leiden collection. Schlegel, who succeeded Temminck as director of the museum in 1858, also described a fair number of new amphibians and reptiles from the Dutch East Indies.

Starting the second half of the $19^{\text {th }}$ century, the Leiden museum maintained an active exchange-policy with other European museums, including Berlin, Frankfurt, Munich, Vienna and Paris. Sometimes the exchange comprised specimens belonging to type series, and as a result the type material is scattered over different museums. The exchange policy brought to Leiden type specimens described by J. B. von Spix, W. Peters and prince Wied-Nieuwied, among others; it continued during the $20^{\text {th }}$ century, further enriching the amphibian collection.

Throughout the first half of the $20^{\text {th }}$ century, museum expeditions to collect and study the fauna of the Dutch colonies continued to be organized, including several multidisciplinary expeditions sent to Suriname and Indonesia by the Koninklijk Nederlands Aardrijkskundig Genootschap (or KNAG, the Royal Dutch Geographical Society). The KNAG expeditions to map Suriname's rivers and to explore unknown territories of Dutch New Guinea (now Papua, Indonesia) made significant geological, zoological, botanical and ethnological collections. Hilbrand Boschma and Leo Daniël Brongersma, both herpetologists and successively directors of the Leiden museum, participated in these expeditions. The zoological material is deposited in Leiden and in the Zoölogisch Museum Amsterdam.

During the second half of the century, the Herpetology department of the RMNH shifted its focus from Indonesia to Surinam and the Amazonian region, mostly due to the efforts of Marinus S. Hoogmoed, curator at the museum from 1963 until 2003. Hoogmoed participated in several expeditions to Suriname and Brazil, that resulted in the discovery of unknown amphibian species and an important herpetological collection. Hoogmoed's role as curator of the herpetological collection has been a crucial one, since he ordered part of the collection, discovered many type specimens from the older jars, selected lectotypes and described several new species.

Given the large number of species that have been described on the basis of the Leiden collection, the RMNH type collection is of great scientific significance. This catalogue is an attempt to locate and index all type material housed in the Leiden amphibian collection. This is not an easy task, especially when it concerns taxa described in the first half of the $19^{\text {th }}$ century, when type material was not always labeled as such. More- 
over, the original publications rarely identified the specimens used for the description or the collection housing them, and museum records from that time often provide incomplete information. We therefore invite colleagues to provide corrections and additions wherever possible, with the purpose of attaining a more complete catalogue of the amphibian type specimens in the RMNH.

\section{How to use this catalogue}

This catalogue is alphabetically ordered on the original species name, followed by author, year of publication and pagination. The full reference can be found in the references section. Current scientific names are given. The synonymy follows Frost (2006).

The information provided is based on the original publications. Pagination, figures and plates are also given. If the original description was not available, the information is derived from museum records and labels. Specimens that possibly belong to a type series are also listed, with a special indication that the type status could not be confirmed. The location of other types of a particular series in other institutions is given when known, but was not actively researched. Sometimes, the year in which a specimen arrived at the RMNH can be relevant to settle type-status questions. In those cases, the date is given as e.g. "Received: 1862".

Quotation marks are used to indicate that the type locality is directly taken from the original description; otherwise the information is extracted from the RMNH labels and records. When detail to a locality is added, square brackets are used, for example: "Hatam" [Arfak Mountains, Irian Jaya, Indonesia]. When a locality name has been changed, square brackets are also used to give the current name of the particular locality: "Nova Guinea" [Irian Jaya, Indonesia]. In a few cases the data from the description differ from the data in the RMNH records or the label. An account of the inconsistency is then given under "Remarks". Status and taxonomical problems are also given under "Remarks". For a discussion on particular issues, the reader is referred to Frost (2006). Unless otherwise noted, the material is preserved in alcohol (70\% ethyl alcohol). When a specimen is damaged or in poor condition, this is also indicated under "Remarks".

New names and new combinations for the original names are alfabetically listed in the Appendix.
Abbreviations
Ad. adult
Don. donator
Ex the specimen was obtained from another institution, purchased from a dealer or obtained by donation
Gen. genus
Hgr. half grown
Juv. juvenile
Leg. legit or collector
Loc. type locality
Nov. novum or new
Sp. species
Spec. $\quad \operatorname{specimen}(\mathrm{s})$

\section{Museum abbreviations}

AMNH American Museum of Natural History in New York, USA.

BM Natural History Museum in London, UK (formerly the British Museum [Natural History]) 
CJG Coleman J. Goin Collection, now in the Museum of Northern Arizona in Flagstaff, Arizona, USA.

CM Carnegie Museum in Pittsburgh, Pennsylvania, USA.

FMNH Field Museum of Natural History in Chicago, Illinois, USA.

SMF Forschungsinstitut und Natur-Museum Senckenberg in Frankfurt, Germany.

FSM Florida State Museum, University of Florida, USA.

IRSNB Institue Royal des Sciences Naturelles de Belgique in Brussels, Belgium (formerly the Muséum Royal d'Histoire Naturelle, Belgium)

KU Museum of Natural History, University of Kansas in Lawrence, Kansas, USA.

MCZ Museum of Comparative Zoology in Cambridge, Massachusetts, USA.

MHNG Muséum d'Histoire Naturelle in Geneva, Switzerland.

MNHNP Muséum National d'Histoire Naturelle in Paris, France.

MSNG Museo Civico di Storia Naturale "Giacomo Doria" in Genova, Italy.

MPEG Museu Paraense Emilio Goeldi in Belém, Brazil.

KBIN Koninklijk Belgisch Instituut voor Natuurwetenschappen in Brussels, Belgium.

MB Museu Bocage, Universidade de Lisboa, Portugal.

MNCN Museo Nacional de Ciencias Naturales in Madrid, Spain.

MZUF Museo Zoologico La Specola in Florence, Italy.

MZUT Museo di Zoologia Universitá di Torino, Italy.

NHMW Naturhistorisches Museum Wien in Vienna, Austria.

PM National Museum and Art Gallery in Port Moresby, Papua New Guinea.

QCAZ Museo de Zoología, Pontificia Universidad Católica del Ecuador in Quito, Ecuador.

RMNH National Museum of Natural History in Leiden, The Netherlands.

SAM South African Museum in Cape Town, South Africa.

SAMA South Australian Museum in Adelaide, Australia.

UFC Universidad Federal do Ceará in Fortaleza, Brazil.

UMMZ University of Michigan Museum of Zoology, Michigan, USA.

UP University of Papua New Guinea, Biology Department in Port Moresby, Papua New Guinea.

USNM National Museum of Natural History in Washington, USA.

UTA University of Texas at Arlington, USA.

WCAB Bokermann Collection, now in the Museu de Zoologia Universidade de São Paulo.

ZMA Zoölogisch Museum Amsterdam, The Netherlands.

ZMB Museum für Naturkunde der Humboldt-Universität zu Berlin, Germany.

ZMH Zoologisches Museum in Hamburg, Germany.

ZMU Zoologisk Museum in Copenhagen, Denmark.

ZSM Zoologische Staatssammlung München in Munich, Germany.

\section{Acknowledgements}

We thank D.R. Frost (New York, USA), M.S. Hoogmoed (Belém, Brazil) and J.P. Michels (Amsterdam, The Netherlands) for their advice and valuable contributions.

The registration of types in the amphibian collection was partly financed by the "Delta Plan for the Preservation of Cultural Heritage" (the Deltaplan voor Cultuurbehoud), issued by the former Ministry of Welfare, Public Health and Culture in 1991. 


\section{LIST OF TYPE SPECIMENS}

\section{Adelophryne adiastola Hoogmoed \& Lescure, 1984}

Hoogmoed \& Lescure, 1984. Zool. Meded., Leiden, 58: 95; figs. 4 -7, 11.

Adelophryne gen. nov. Hoogmoed \& Lescure, 1984. Zool. Meded., Leiden, 58: 95.

Paratype: RMNH 21746 (formerly UTA 4941), 1 ox. Loc.: "Yapima, Vaupés River, Vaupés, Colombia". Leg.: W.F. Pyburn. Ex: University of Texas at Arlington.

Remarks. The holotype and two paratypes are in the Vertebrate Collection of the University of Texas at Arlington (UTA 4943 and UTA 4940, 4942).

\section{Adelophryne baturitensis Hoogmoed, Borges \& Cascon, 1994}

Hoogmoed, Borges \& Cascon, 1994. Zool. Meded., Leiden, 68: 280; figs. 10-11.

Paratypes: RMNH 26662 (formerly UFC 2656), 1 ㅇ. Loc: "Sítio Barbosa, Serra de Baturité, Município de Pacoti, CEARÁ, Brasil”. Leg.: D.M. Borges.

RMNH 26663 (formerly UFC 2663), 1 spec. Loc.: "Sítio Floresta, Serra de Baturité, Município de Pacoti, CEARÁ, Brasil”. Leg.: D.M. Borges.

RMNH 26664-26665 (formerly UFC 2668 and UFC 2670), 2 spec. Loc.: "Sítio Timbaúba e Sítio Sâo Pedro, Serra de Baturité, Município de Pacoti, CEARÁ, Brasil”. Leg.: D.M. Borges.

Remarks. The holotype (UFC 2655) and several paratypes are in the Universidad Federal do Ceará in Fortaleza, Brazil. Other paratypes are in the Museu Paraense Emilio Goeldi in Belém, Brazil.

\section{Adelophryne maranguapensis Hoogmoed, Borges \& Cascon, 1994}

Hoogmoed, Borges \& Cascon, 1994. Zool. Meded., Leiden, 68: 289; figs. 12-16.

Paratype: RMNH 26666 (formerly UFC 2676), 1 spec. Loc.: "Brasil. Ceará. Municipio de Maranguape. Serra de Maranguape, Pico da Rajada, 890 m.”. Leg.: W. Franklin-Júnior and N. Salgado.

Remarks. The holotype (UFC 2672) and five paratypes are in the Universidad Federal do Ceará in Fortaleza, Brazil.

\section{Andinophryne colomai Hoogmoed, 1985}

Hoogmoed, 1985. Zool. Meded. Leiden, 59: 264; figs. 3-7, 10-12.

Andinophryne gen. nov. Hoogmoed, 1985. Zool. Meded. Leiden, 59: 254.

Holotype: RMNH 21905, 1 ․ Loc.: "Cabeceras del Rio Baboso, cerca a Lita, Carchi Province, Ecuador”. Leg.: L. Coloma and A. Yépez.

\section{Ansonia latidisca Inger, 1966}

Inger, 1966. Fieldiana, Zool., 52: 108.

Holotype: RMNH 10677, 1 ơ. Loc.: “top of Mount Damus, Sambas, Kalimantan” [Indonesia]. Leg.: J.G. Hallier. 


\section{Ansonia longidigita gryllivoca Inger, 1960}

Inger, 1960. Fieldiana, Zool., 39: 483.

Current name: Ansonia leptopus (Günther, 1872)

Paratype: RMNH 10486 (formerly FMNH 77417), 1 spec. Loc.: "in primary forest in the Sungei Tawan, a small tributary of the Kalabakan River, Tawau District, North Borneo [Sabah]" [Malaysia]. Leg.: R.F. Inger.

Remarks. The holotype (FMNH 77397) is in the Field Museum of Natural History in Chicago.

\section{Asterophrys similis Zweifel, 1956}

Zweifel, 1956. Am. Mus. Novit., 1766: 5; fig. 2.

Current name: Xenorhina similis (Zweifel, 1956)

Paratype: RMNH 10470 (formerly AMNH 43748), 1 spec. Loc.: "Bele River, 18 km. north of Lake Habbema, 2200 m., Netherlands New Guinea" [Irian Jaya, Indonesia]. Leg.: Archbold Expedition. Ex: American Museum of Natural History in New York.

Remarks. Type specimens are also in the American Museum of Natural History in New York (holotype: AMNH A43722; paratypes: AMNH A43725-27, 43721, 43723, 43730, 43749-56, 43758, 43760).

\section{Atelopus flavescens Duméril \& Bibron, 1841}

Duméril \& Bibron, 1841. Erp. Gén., 8: 661.

Paralectotypes: RMNH 2208, 2 spec. Loc.: "Amérique méridional (...) sur les bords d'un ruisseau, dans une montagne près de Cayenne" [French Guyana]. Ex: Muséum Nationale d'Histoire Naturelle in Paris. Received: 1844.

Remarks. The lectotype was designated by Lescure (1976: 478) and it is deposited in the Muséum National d'Histoire Naturelle in Paris (MNHNP 803). There are two paralectotypes in that same collection (MNHNP 256).

\section{Atelopus spumarius barbotini Lescure, 1981}

Lescure, 1981. Bull. Mus. Natl. Hist. Nat. Paris, Ser. 4(A), 3: 896.

Current name: Atelopus spumarius Cope, 1871

Paratype: RMNH 20357, 1 o`. Loc.: "10 km. au nord-est du Saint-Laurent" [French Guyana]. Leg.: M.S. Hoogmoed and W.N. Polder.

Remarks. The holotype is in the Muséum National d'Histoire Naturelle in Paris (MNHNP 1979-1505).

\section{Aubria occidentalis Perret, 1994}

Perret, 1994. Tropical Zool., 7: 258; figs. 1-9.

Paratypes: RMNH 6525, 1 ㅇ. Loc.: "Liberia, Hilltown”. Leg.: J. Büttikofer and F.X. Stampfli.

RMNH 1948 A-E, 5 o $^{\star}{ }^{x}$. Loc.: Dabocrom, "Ghana”. Leg.: H.S. Pel.

RMNH 1948 F, 1 ㅇ. Loc.: Dabocrom, "Ghana”. Leg.: H.S. Pel.

Remarks. The holotype is in the Museum d'Histoire Naturelle in Geneva (MHNG 2129.17). Paratypes are also in the Museum d'Histoire Naturelle in Geneva (MHNG 1189.19-20, 1558.47, 2129.15-16, 2129.18, 1522.28), in the Museo Zoologico La Specola in Florence (MZUF 16473) and the Zoologisk Museum in Copenhagen (ZMUC 0171190, 037364, 07856). 


\section{Batrachylodes elegans Brown \& Parker, 1970}

Brown \& Parker, 1970. Breviora, 346: 14.

Paratypes: RMNH 17172 (formerly MCZ), 7 spec. Loc.: "Mutahi on Bougainville Island, Solomon Islands". Leg.: F. Parker.

RMNH 17174 (formerly MCZ), 20 spec. Loc.: "Mutahi on Bougainville Island, Solomon Islands". Leg.: F. Parker.

Remarks. The holotype as well as paratypes are in the Museum of Comparative Zoology in Cambridge, Massachusetts (MCZ 54559).

\section{Bombinator baleatus Müller in Oort \& Müller, 1833}

Müller in Oort \& Müller, 1833. Verh. Batav. Genootsch. Wetensch., 16: 96.

Current name: Kaloula baleata (Müller In Oort \& Müller, 1833)

Syntype: RMNH 2218, 1 spec. Loc.: "Krawang" [Java, Indonesia]. Leg.: S. Müller.

Remarks. Parker (1934: 89) indicated that BM 44.2.22.93 (2 $0^{x} 0^{x}$ and 1 ㅇ from Java, in the Natural History Museum in London; exanged from the National Museum of Natural History in Leiden) might be part of the type series. RMNH 2218 could not be located in the Leiden collection.

\section{Bombinator oxycephalus Schlegel, 1858}

Schlegel, 1858. Handl. Dierk., 2: 58.

Current name: Xenorhina oxycephala (Schlegel, 1858)

Syntypes: RMNH 2280, 2 spec. Loc.: "Nieuw-Guinea” [Irian Jaya, Indonesia]. Leg.: S. Müller. Remarks. Zweifel (1972a: 536) restricted the type locality to "Triton Bay, West Irian".

\section{Boulengerula taitanus Loveridge, 1935}

Loveridge, 1935. Bull. Mus. Comp. Zool., 79: 16.

Current name: Boulengerula taitana Loveridge, 1935

Paratype: RMNH 6436 B (formerly MCZ 20024), 1 spec. Loc.: "Mt. Mbobolo, Taita Mts., Kenya". Ex: Museum of Comparative Zoology.

Remarks. Originally there were two specimens in RMNH 6436. One of these (RMNH 6436 A, formerly MCZ 20016) seems to have been lost. The holotype and paratypes are in the Museum of Comparative Zoology in Cambridge, Massachusetts (MCZ 20001 and MCZ 20002-20015, 20017-20023, respectively).

\section{Bufo albicans Spix, 1824}

Spix, 1824. Animal. Nova Spec. Nov. Test. Ran. Brasil: 47; pl. XVIII, fig. 2.

Current name: Chaunus marinus (Linnaeus, 1758)

Paralectotype: RMNH 2191, 1 spec. Loc.: "flumen Nigrum” [Rio Negro, Brazil]. Leg.: J.B. von Spix.

Remarks. The lectotype was selected by Hoogmoed \& Gruber (1983: 371) and it is deposited in the Zoologische Staatssammlung München (ZSM 1140/0).

\section{Bufo albifrons Spix, 1824}

Spix, 1824. Nova Spec. Nov. Test. Ran. Brasil: 48; pl. XIX, fig. 2.

Current name: Physalaemus albifrons (Spix, 1824) 
Lectotype: RMNH 2272, 1 ㅇ․ Loc.: "Habitat in Provincia Bahia" [Brazil]. Leg.: J.B. von Spix.

Remarks. Hoogmoed \& Gruber (1973: 374) stated that the two original syntypes of this species in the Zoologisches Staatssammlung München (ZSM 49/0 and 50/0) should be considered lost. Subsequently Hoogmoed (1986: 299) considered RMNH 2272 the remaining syntype of the original series and designated it as lectotype. The type locality was given as "Porto Alegre" [Brazil] by Boulenger (1887: 296). According to Bokermann (1966a: 17) the type locality is "provàvelmente . . . da região central de taboleiros" [Bahia, Brazil].

\section{Bufo asper Gravenhorst, 1829}

Gravenhorst, 1829. Delic. Mus. Zool. Vratislav., 1: 58.

Current name: Phrynoidis aspera (Granvenhorst, 1829)

Lectotype: RMNH 2172, 1 spec. Loc.: "Java" [Indonesia]. Leg.: H. Kuhl and J.C. van Hasselt.

Paralectotypes: RMNH 10681 (formerly RMNH 2172), 3 spec. Loc.: "Java" [Indonesia]. Leg.: H. Kuhl and J.C. van Hasselt.

Remarks. The lectotype was designated by Inger (1966: 66).

\section{Bufo brongersmai Hoogmoed, 1972}

Hoogmoed, 1972. Zool. Meded. Leiden, 47: 50; fig. 1-3, pl. I, II.

Current name: Pseudepidalea brongersmai (Hoogmoed, 1972)

Holotype: RMNH 16782, 1 ㅇ. Loc.: “10 km SW. of Tiznit, along road Tiznit-Mirhleft, Morocco”. Leg.: M.S. Hoogmoed.

Paratypes: RMNH 16783, 2 o $^{\star}$. Loc.: "Souk-el-Arba-du-Sahel, $25 \mathrm{~km} \mathrm{SW}$. of Tiznit, along road Tiznit-Mirhleft, Morocco". Leg.: M.S. Hoogmoed.

RMNH 16784, 2 ơ ox; RMNH 16804, 23 larvae. Loc.: “Oued Seyad, 4 km E. of Fask, Morocco”. Leg.: M.S. Hoogmoed.

RMNH 16785, 6 juv. Loc.: "Tafraoute, Anti-Atlas, Morocco". Leg.: M.S. Hoogmoed.

RMNH 16786, 1 ㅇ․ Loc.: "Vallée des Ammeln, N. of Tafraoute, Anti-Atlas, Morocco". Leg.: M.S. Hoogmoed.

Remarks. There is also a paratype in the Naturhistorisches Museum Wien in Vienna (NHMW 17221).

\section{Bufo cruentatus Tschudi, 1838}

Tschudi, 1838. Classif. Batr.: 52, 88.

Current name: Leptophryne cruentata (Tschudi, 1838)

Syntypes: RMNH 2130, 2 spec. Loc.: "India orient." [Indonesia]. Leg.: H. Boie and H.C. Macklot. RMNH 2131, 1 spec. Loc.: "India orient." [Indonesia]. Leg.: H. Kuhl and J.C. van Hasselt.

Remarks. The type locality in Tschudi (1838: 88) is "India orient.", restricted to "Java" [Indonesia] by Duméril \& Bibron (1841: 666). RMNH 2131 could not be located in the Leiden museum.

\section{Bufo dorsalis Spix, 1824}

Spix, 1824. Nova Spec. Nov. Test. Ran. Brasil : 46; pl. XVII, fig. 2.

Current name: Chaunus ornatus (Spix, 1824)

Paralectotype: RMNH 2189, 1 ơ. Loc.: "Provincia Rio de Janeiro" [Brazil]. Leg.: J.B. von Spix.

Remarks. The lectotype and a paralectotype are in the Zoologische Staatssammlung München in Munich (ZSM 1141/0 A and B, respectively). The lectotype was selected by Hoogmoed \& Gruber (1983: 373). 
Holotype: RMNH 10867, 1 ๙ . Loc.: "Porlamar, Isla Margarita, Venezuela". Leg.: P. Wagenaar Hummelinck. Paratypes: RMNH 10868, $9{\sigma^{\star}}^{\star}, 1$ 우. Loc.: "Porlamar, Isla Margarita, Venezuela". Leg.: P. Wagenaar Hummelinck.

\section{Bufo gymnauchen Bleeker, 1858}

Bleeker, 1858. Natuurkd. Tijdschr. Nederl. Indië, 16: 46.

Current name: Duttaphrynus melanostictus (Schneider, 1799)

Paralectotype: RMNH 3961, 1 spec. Loc.: "Indischen archipel” [Indonesia]. Ex: Bleeker Collection.

Remarks. Boulenger (1882: 307) designated BM 1947.2.21.71 (in the Natural History Museum in London) as lectotype by implication. The type locality was restricted to "Bintang" [Pulau Bintang, Roux Archipelago] by Boulenger (1882: 307). See also Inger (1966: 70).

\section{Bufo ictericus Spix, 1824}

Spix, 1824. Nova Spec. Nov. Test. Ran. Brasil: 44; pl. XVI, fig. 1.

Current name: Chaunus ictericus (Spix, 1824)

Lectotype: RMNH 2182, 1 spec. Loc.: "Provinciae Rio de Janeiro" [Brazil]. Leg.: J.B. von Spix. Remarks. The lectotype was designated by Hoogmoed \& Gruber (1983: 372).

\section{Bufo juxtasper Inger, 1964}

Inger, 1964. Fieldiana, Zool., 44: 154.

Current name: Phrynoidis juxtaspera (Inger, 1964)

Paratypes: RMNH 14515, 1 spec. Loc.: “upper Sibau River, Kalimantan” [Indonesia]. Leg.: J. Büttikofer. RMNH 14517, 1 spec. Loc.: "upper Mahakam River, Kalimantan” [Indonesia]. Leg.: A.W. Nieuwenhuis. Remarks. The RMNH paratypes were numbered after Inger's publication. The holotype (FMNH 77472) and paratypes are in the Field Museum of Natural History in Chicago.

\section{Bufo mauritanicus Schlegel, 1841}

Schlegel, 1841. In Wagner (ed.), Reisen Regentschaft Algier, 3: 134.

Holotype: RMNH 2122, 1 spec. Loc.: “...in den Umgebungen der Stadt Algier... Sümpfen der Ebene Metidscha; ... Bona” [Algeria]. Leg.: M.F. Wagner.

\section{Bufo ornatus Spix, 1824}

Spix, 1824. Nova Spec. Nov. Test. Ran. Brasil: 45; pl. XVI, fig. 2.

Current name: Chaunus ornatus (Spix, 1824)

Paralectotype: RMNH 2157, 1 hgr. Loc.: "Provincia Rio de Janeiro" [Brazil]. Leg.: J.B. von Spix.

Remarks. The lectotype (ZSM 2691) was designated by Hoogmoed \& Gruber (1983: 373) and is in the Zoologische Staatssammlung München in Munich. 


\section{Bufo Spixii Fitzinger, 1826}

Fitzinger, 1826. Neue Class. Rept.: 65.

Current name: Chaunus ornatus (Spix, 1824)

Paralectotype: RMNH 2190, 1 spec. Loc.: "Provincia Rio de Janeiro" [Brazil]. Leg.: J.B. von Spix.

Remarks. The lectotype (ZSM 1343/0) was designated by Hoogmoed \& Gruber (1983: 373) and is in the Zoologische Staatssammlung München, together with paralectotypes (ZSM 1343/0).

\section{Bufo vulgaris var. japonicus Temminck \& Schlegel, 1838}

Temminck \& Schlegel, 1838. Fauna Japonica, 3: 106; pl. II, fig. 5, 6.

Current name: Bufo japonicus Temminck \& Schlegel, 1838

Lectotype: RMNH 2119 A, 1 spec. Loc.: “Japon”. Leg.: Ph.F. von Siebold.

Paralectotypes: RMNH 2119 B, 1 spec. Loc.: "Japon”. Leg.: Ph.F. von Siebold.

RMNH 2109, 6 spec.; RMNH 2115, 3 spec. Loc.: "Japon”. Leg.: Ph.F. von Siebold and H. Bürger.

RMNH 2116, 2 spec.; RMNH 2117, 4 spec. Loc.: "Japon”. Leg.: H. Bürger.

Remarks. The lectotype was designated by Matsui (1984: 407).

\section{Caecilia annulata Wagler, 1824}

Wagler, 1824. Serp. Brasil. Spec. Nov. Hist. Nat. Esp. Nouv. Serpens: 74; pl. XXVI, fig. 1.

Current name: Siphonops annulatus (Mikan, 1820)

Lectotype: RMNH 2419, 1 spec. Loc.: "provinciae Bahiae" [Brazil]. Leg.: J.B. von Spix.

Remarks. Hoogmoed \& Gruber (1983: 342) concluded that only one of Wagler's syntypes is still preserved (RMNH 2419) and selected it as lectotype of the species.

\section{Caecilia oxyura Duméril \& Bibron, 1841}

Duméril \& Bibron, 1841. Erp. Gén., 8: 280.

Current name: Uraeotyphlus oxyurus (Duméril \& Bibron, 1841).

Paralectotype: RMNH 2414, 1 spec. Loc.: "Côte du Malabar" [India]. Leg.: J.J. Dussumier. Ex: Muséum National d'Histoire Naturelle in Paris.

Remarks. According to Guibé (1950: 4) the type series consists of RMNH 2414 and MNHNP 4271 (2 spec., in the Muséum National d'Histoire Naturelle in Paris). Lescure (1988: 61) designated MNHNP 4271 B as lectotype.

\section{Callula frontifasciata Horst, 1883}

Horst, 1883. Notes Leyden Mus., 5: 243.

Current name: Oreophryne frontifasciata (Horst, 1883)

Lectotype: RMNH 1807, 1 spec. Loc.: "Morotai” [Indonesia]. Leg.: H.A. Bernstein.

Paralectotype: RMNH 8686, 1 spec. Loc.: "Morotai" [Indonesia]. Leg.: H.A. Bernstein.

Remarks. The lectotype was selected by Brongersma (1948: 308). Horst (1883: 243) referred to "several specimens from Selawatti, Morotai and Halmaheira, collected by Bernstein". The specimens from Selawatti and Halmaheira could not be located in the Leiden museum.

\section{Centrolenella geijskesi Goin, 1966}

Goin, 1966b. Stud. Fauna Suriname and other Guyanas, 8: 77; fig. 102.

Current name: Cochranella geijskesi (Goin, 1966) 
Holotype: RMNH 11041, 1 ㅇ․ Loc.: "at about 200 meters altitude on the south slope of the Wilhelmina Mountains, District Nickerie, Suriname". Leg.: S. Ligorie.

\section{Ceratophryne nasuta Schlegel, 1858}

Schlegel, 1858. Handl. Dierkd., 2: 57; pl. IV, fig. 72.

Current name: Megophrys nasuta (Schlegel, 1858)

Syntypes: RMNH 2143, 2 spec. Loc.: "Patang-Singalang, Sumatra" [Batang Singalang, Sumatra, Indonesia]. Leg.: S. Müller.

\section{Ceratophrys Boiei Wied-Neuwied, 1824}

Wied-Neuwied, 1824. Isis v. Oken, 14: 673.

Current name: Proceratophrys boiei (Wied-Neuwied, 1824)

Lectotype: RMNH 24109 (formerly RMNH 2151), 1 spec. Loc.: "Brasil”. Leg.: J. Natterer.

Remarks. Hoogmoed (1990: 269) designated RMNH 24109 as lectotype. A paralectotype is probably in the Museum für Naturkunde der Humboldt-Universität zu Berlin. The type locality was given as "Bahia" and "Rio de Janeiro" by Wied-Neuwied (1825: 592). Bokermann (1966a: 25) restricted the type locality to "Rio de Janeiro, Guanabara".

\section{Ceratophrys turpicola Schlegel, 1837}

Schlegel, 1837-1844. Abbild. Neuer Unvollst. Amph., 1: 30; pl. X, fig. 4.

Current name: Asterophrys turpicola (Schlegel, 1837)

Syntypes: RMNH 2153, 2 ơox. Loc.: "Westküste von Neu-Guinea" [Irian Jaya, Indonesia]. Leg.: S. Müller and H.C. Macklot.

Remarks. Brongersma (1953: 573) restricted the type locality to "Lobo district, Triton Bay, Dutch New Guinea". Though the publication date on the first page of Schlegel's Abbildungen is 1837-1844, the work was issued in parts. See Stejneger's Herpetology of Japan (1907: 540) for the publication dates.

\section{Chaperina basipalmata Van Kampen, 1906}

Van Kampen, 1906. Nova Guinea, 5: 169; figs. 4, 5.

Current name: Austrochaperina basipalmata (Van Kampen, 1906)

Syntypes: RMNH 4628, 1 spec. Loc.: "Timena-Fluss" [Irian Jaya, Indonesia]. Leg.: Nieuw Guinea Expeditie, 1903.

RMNH 4629, 1 juv. Loc.: "Tawarin" [Irian Jaya, Indonesia]. Leg.: Nieuw Guinea Expeditie, 1903.

Remarks. According to Van Tuijl (1995), other syntypes are in the Zoological Museum Amsterdam (ZMA 5875-5876).

\section{Chaperina macrorhyncha Van Kampen, 1906}

Van Kampen, 1906. Nova Guinea, 5: 168; fig. 3.

Current name: Austrochaperina macrorhyncha (Van Kampen, 1906)

Holotype: RMNH 4630, 1 spec. Loc.: "Manikion-Gebiet" [Irian Jaya, Indonesia]. Leg.: Nieuw Guinea Expeditie, 1903. 


\section{Chioglossa lusitanica longipes Arntzen \& Alexandrino, in press}

Arntzen \& Alexandrino In Arntzen, Groenenberg, Alexandrino, Sequeira \& Ferrand, in press. Journal of Natural History.

Holotype: RMNH 40113, 1 ơ. Loc.: "Mina das Águas Férreas, Serra de Santa Justa, Valongo, N 4111'18.3' and W8 29' 18.4'; Valongo, Douro Litoral in north-western Portugal”. Leg. : F. Sequeira and J.W. Arntzen.

Paratypes: RMNH 18796-18805, 10 specs. Loc.: "Ribeira da Silveirinha, Serra de Santa Justa, Valongo, N

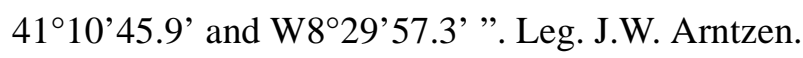

RMNH 40114-40116, 3 specs. Loc.: "Mina das Águas Férreas, Serra de Santa Justa, Valongo, N 41 ${ }^{\circ} 11^{\prime} 18.3^{\prime}$ and W8 ${ }^{\circ} 9^{\prime} 18.4^{\prime}$; Valongo, Douro Litoral in north-western Portugal'. Leg. : F. Sequeira and J.W. Arntzen.

Remarks. Other paratypes are in the Museo Bocage in Lisbon (MB-06-450-01 - MB-06-450-04); the Museo Nacional de Ciencias Naturales in Madrid (MNCN 43641-43644) and the Zoölogisch Museum Amsterdam (ZMA 7307a-e, ZMA 7340a-j, ZMA 7358 a-d, ZMA 7387 a-d, ZMA 7402, ZMA 7482, ZMA 7588a-c, ZMA 7669 and ZMA 8086a-k).

\section{Chiroleptes inermis Peters, 1867}

Peters, 1867. Monatsber. Preuss. Akad. Wiss. Berlin: 30.

Current name: Litoria inermis (Peters, 1867)

Syntype: possibly RMNH 1888, 1 spec. Loc.: Rockhampton, Australia. Ex: Museum Godeffroy, Hamburg.

Remarks. Other types are in the American Museum of Natural History in New York (AMNH 23582) and the Naturhistorische Museum Wien in Vienna (NHMW 18384, 2 spec.). Cogger et al. (1983: 44) regarded ZMB 5589 (3 specimens, in the Museum für Naturkunde der Humboldt-Universität zu Berlin) as the holotype of the species and considered the status of other syntypes as "apparently in error". However, Peters (1867:30) did mention several specimens from the Godeffroy Museum for his description: "Andere Exemplare ebendaher in der Godeffroyschen Sammlung." If Peters indeed included those in his decriptions, RMNH 1888 could be a syntype.

\section{Coecilia hypocyanea Boie, 1827}

Boie, 1827. Isis v. Oken, 1827: 565.

Current name: Ichthyophis hypocyaneus (Boie, 1827)

Paratypes: RMNH 2408, 1 ad. Loc.: "Java” [Indonesia]. Leg.: H. Kuhl and J.C. van Hasselt. RMNH 2409, 1 larva. Loc.: "Java" [Indonesia].

Remarks. Häupl, Tiedemann, \& Grillistsch (1994: 11) catalogued NHMW 9097 (in the Naturhistorisches Museum Wien in Vienna, exchanged from the National Museum of Natural History in Leiden in 1825) as the holotype. However, RMNH 2408, 2409 and NHMW 9097 are all part of the original type series. See also Taylor (1968: 97). The type locality ("Java") is restriced to "wet and marshy places on the north coast of Bantam (Province), western Java” by Taylor (1968: 97).

\section{Colostethus toachi Coloma, 1995}

Coloma, 1995. Misc. Publ. Mus. Nat. Hist. Univ. Kansas, 87: 52.

Current name: Hyloxalus toachi (Coloma, 1995)

Paratypes: RMNH 25248-25250, 3 spec. Loc: "Finca Gloria, 15 km NW Alluriquín (5 km NW of La Florida), Provincia Pichincha, Ecuador". Leg.: M.S. Hoogmoed and L.A. Coloma.

Remarks. The holotype is in the Museo de Zoología, Pontificia Universidad Católica del Ecuador in Quito (QCAZ 1437). 


\section{Cornufer unicolor Tschudi, 1838}

Tschudi, 1838. Classif. Batr.: 28, 71.

Current name: Pelorius nortoni (Schwartz, 1976)

Paralectotype: RMNH 1736, 1 spec. Loc.: "Nova Guinea” [Irian Jaya, Indonesia]. Leg.: S. Müller.

Remarks. Zweifel (1967: 119) designated MNHNP 747 as lectotype (in the Muséum National d'Histoire Naturelle in Paris).

\section{Dendrobates azureus Hoogmoed, 1969}

Hoogmoed, 1969. Zool. Meded. Leiden, 44: 134; pl. 1, figs 1, 2; pl. 2, figs. 1-6; table I.

Holotype: RMNH 13837 A, 1 spec. Loc.: "Sipaliwini, forest-island on western slope Vier Gebroeders Mountain, $2^{\circ} \mathrm{N} 55^{\circ} 58^{\prime} \mathrm{W}$, Surinam”. Leg.: M.S. Hoogmoed.

Paratypes: RMNH 13837 B-I, 8 spec.; RMNH 13837 K, 1 spec. Loc.: "Sipaliwini, forest-island on western slope Vier Gebroeders Mountain, $2^{\circ} \mathrm{N} 55^{\circ} 58^{\prime} \mathrm{W}$, Surinam”. Leg.: M.S. Hoogmoed.

RMNH 13838, 1 spec. Loc.: "Sipaliwini, forest-island on western slope Vier Gebroeders Mountain, $2^{\circ} \mathrm{N}$ 55 58’ W, Surinam”. Leg.: M.S. Hoogmoed.

RMNH 13839 A-D, 5 spec. Loc.: "Sipaliwini, forest-island $1.5 \mathrm{~km}$ northeast of Vier Gebroeders Mountain, $2^{\circ} 1^{\prime}$ N 55 57’30”'W, Surinam”. Leg.: M.S. Hoogmoed.

RMNH 13840 A-F, 6 spec. Loc.: "Sipaliwini, forest-island $2 \mathrm{~km}$ north of Vier Gebroeders Mountain, $2^{\circ} 1^{\prime} \mathrm{N} 55^{\circ} 58^{\prime} \mathrm{W}$, Surinam”. Leg.: M.S. Hoogmoed.

RMNH 13841 A-C, 3 spec. Loc.: "Sipaliwini, forest-island on northeastern slope of Vier Gebroeders Mountain, $2^{\circ} 1$ 'N 55 $57^{\prime} 30^{\prime \prime}$ W, Surinam”. Leg.: M.S. Hoogmoed.

RMNH 13842, 1 ox, 2 tadpoles. Loc.: "Sipaliwini, forest-island on western slope Vier Gebroeders Mountain, $2^{\circ} \mathrm{N} 55^{\circ} 58^{\prime} \mathrm{W}$, Surinam”. Leg.: M.S. Hoogmoed.

RMNH 13843 A-J, 10 spec. Loc.: "Sipaliwini, forest-island on western slope Vier Gebroeders Mountain, $2^{\circ} \mathrm{N} 55^{\circ} 58^{\prime} \mathrm{W}$, Surinam”. Leg.: M.S. Hoogmoed.

Remarks. One paratype is at the University of Texas at Arlington (UTA A 15873; formerly RMNH 13837 J).

\section{Eleutherodactylus alticola Lynn, 1937}

Lynn, 1937. Herpetologica, 1: 89; fig. 2.

Current name: Euhyas alticola (Lynn, 1937)

Paratypes: RMNH 10035 (formerly USNM 102526-102546), 2 spec. Loc.: "on or near the summit of Blue Mountain Peak, Jamaica". Leg.: W. G. Lynn. Ex: National Museum of Natural History in Washington.

Remarks. The holotype is in the National Museum of Natural History in Washington (USNM 102524).

\section{Eleutherodactylus chiastonotus Lynch \& Hoogmoed, 1977}

Lynch \& Hoogmoed, 1977. Proc. Biol. Soc. Washington, 90: 425; figs. 1-5.

Holotype: RMNH 17614, 1 ơ. Loc.: "Brownsberg, Brokopondo District, Suriname”. Leg.: M.S. Hoogmoed. Paratypes: RMNH 15145, 5 juv. Loc.: "Paloemeu airstrip, Marowijne District, Suriname”. Leg.: M.S. Hoogmoed.

RMNH 17613, 1 ox; RMNH 17615, 1 ox; RMNH 17616, 1 \%, 1 hgr., 1 juv. Loc.: "Brownsberg, 500 m., Brokopondo District, Suriname”. Leg.: M.S. Hoogmoed. 
RMNH 17617, 1 hgr.; RMNH 17619, 1 ơ. Loc.: "4-5 km E of Sipaliwini airstrip, Marowijne District, Suriname". Leg.: M.S. Hoogmoed.

RMNH 17618, 2 juv. Loc.: "Gonini encampment, Coeroeni river, Marowijne District, Suriname”. Leg.: M.S. Hoogmoed.

RMNH 17620, 1 ๙ ${ }^{x}$ Loc.: "Bank Marowijne, opposite Nassau Mts, French Guyana”. Leg.: Suriname Expedition 1948-49.

RMNH 17621, 1 ㅇ RMNH 17622, 1 ㅇ. Loc.: "railway km 121, at foot of Brownsberg, Brokopondo District, Suriname". Leg.: Suriname Expedition 1948-49.

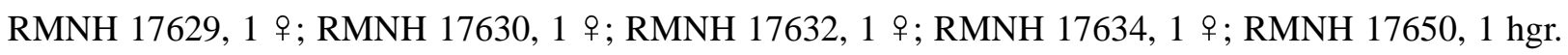
Loc.: "Loë Creek, Camp Hofwijks VII, 54 km S Oelemari airstrip, Marowijne District, Suriname". Leg.: M.S. Hoogmoed and C.W. Myers.

RMNH 17631, 1 우. Loc.: "Loë Creek, Camp Hofwijks VIII, 56 km SSE Oelemari airstrip, Marowijne District, Suriname". Leg.: M.S. Hoogmoed and C.W. Myers.

RMNH 17633, 1 ㅇ. Loc.: "Loë Creek, 2nd Camp, Marowijne District, Suriname”. Leg.: M.S. Hoogmoed and C.W. Myers.

RMNH 17635, 1 ․ Loc.: "5 km SE Camp Hofwijks VII, 59 km S Oelemari airstrip, Marowijne District, Suriname". Leg.: M.S. Hoogmoed.

RMNH 17636, 2 juv. Loc.: "1 km S of Suralco Camp V, 11 km NE airstrip, Marowijne District, Suriname". Leg.: M.S. Hoogmoed and W.N. Polder.

RMNH 17637, 1 hgr., 2 juv.; RMNH 17639, 1 juv.; RMNH 17640, 1 juv.; RMNH 17745, 1 hgr.; RMNH 17746, 1 ơ. Loc.: "Lely Mts., Suralco Camp IV, Marowijne District, Suriname”. Leg.: M.S. Hoogmoed et al.

RMNH 17638, 1 hgr. Loc.: "Loë Creek, Camp Hofwijks IV, Marowijne District, Suriname”. Leg.: M.S. Hoogmoed and C.W. Myers.

RMNH 17641, 2 juv. Loc.: "10 km NE of St. Laurent, French Guyana”. Leg.: M.S. Hoogmoed and W.N. Polder.

RMNH 17642, 1 hgr. Loc.: "6 km S of Blakawatra, Suriname District, Suriname”. Leg.: M.S. Hoogmoed. RMNH 17749, 1 juv. Loc.: "Patamacca, 15 km S main emplacement, Marowijne District, Suriname". Leg.: M.S. Hoogmoed.

\section{Eleutherodactylus lynchi Duellman \& Simmons, 1977}

Duellman \& Simmons, 1977. Proc. Biol. Soc. Washington, 90: 61.

Paratypes: RMNH 17815, 6 spec. Loc.: “Vado Hondo (05²6’ N, 7244’W), 2660 m., Departamento Boyacá, Colombia”. Leg.: W.E. Duellman, D.K. Duellman, J.E. Simmons and L. Trueb.

Remarks. The holotype is in the Museum of Natural History, University of Kansas in Lawrence (KU 168139).

\section{Eleutherodactylus pinarensis Dunn, 1926}

Dunn, 1926. Occas. Pap. Boston Soc. Nat. Hist., 5: 213.

Current name: Euhyas pinarensis (Dunn, 1926)

Paratype: RMNH 9981 (formerly USNM 54399), 1 spec. Loc.: "Rangel, Cuba". Leg.: Henderson and Bartsch. Ex: National Museum of Natural History in Washington.

Remarks. The holotype is in the Museum of Comparative Zoology in Cambridge, Massachusetts (MCZ 3814). 


\section{Eucnemis seychellensis Tschudi, 1838}

Tschudi, 1838. Classif. Batr.: 35, 76.

Current name: Tachycnemis seychellensis (Duméril and Bibron, 1841)

Syntype: RMNH 1767, 1 ex. Loc.: “Seychelles”. Leg.: F. Péron.

Remarks. A syntype is MNHNP 4592, according to Roux-Estève (1979: 26). Tschudi (1838: 35) examined several specimens collected by Péron in Seychelles: "Zuerst brachten Péron und Lesueur von Seychelles alte, hernach Quoy und Gaimard junge Exemplare dieser Species angehöriger Thiere von Madagascar". Therefore we consider RMNH 1767 as part of the type series of Eucnemis seychellensis Tschudi, 1838.

\section{Eleutherodactylus zeuctotylus Lynch \& Hoogmoed, 1977}

Lynch \& Hoogmoed, 1977. Proc. Biol. Soc. Washington, 90: 432; figs. 1, 3, 5-7.

Holotype: RMNH 17701, 1 ơ. Loc.: "west slope, Vier Gebroeders Mt., Sipaliwini, Nickerie District, Suriname". Leg.: M.S. Hoogmoed.

Paratypes: RMNH 15168, 2 spec.; RMNH 15180, 1 spec.; RMNH 15196, 1 spec.; RMNH 17675-17677, 3 spec.; RMNH 17681, 1 hgr., 1 juv. Loc.: "Sipaliwini, Vier Gebroeders Mt., Nickerie District, Suriname". Leg.: M.S. Hoogmoed.

RMNH 17643, 7 hgr. Loc.: "Tafelberg airstrip, Saramacca District, Suriname”. Leg.: M.S. Hoogmoed.

RMNH 17644, 2 우, 1 juv.; RMNH 17646, 1 ơ, 1 우, 3 hgr., 7 juv.; RMNH 17656, 1 우 RMNH 17708, 1 juv.; RMNH 17758 -17765, 17 spec.; RMNH 17770, 1 hgr. Loc.: "Lely mts., Suralco camp IV, Marowijne District, Suriname". Leg.: M.S. Hoogmoed et al.

RMNH 17645, 1 hgr.; RMNH 17648, 1 spec. Loc.: "Lely mts., headquarters of Djoeka creek, Suralco camp V, Marowijne District, Suriname". Leg.: M.S. Hoogmoed.

RMNH 17647, 2 hgr. 우; RMNH 17650, 1 hgr.; RMNH 17654, 1 ; RMNH 17655, 1 우 RMNH 17659, 2 우우. Loc.: "Loë creek, camp Hofwijks VII, 54 km S Oelemari airstrip, Marowijne Distrtict, Suriname". Leg.: M.S. Hoogmoed et al.

RMNH 17649, 3 juv.; RMNH 17768, 2 hgr. Loc.: "Avanavero road, km 251, Paris Jacob Creek, Nickerie District, Suriname". Leg.: M.S. Hoogmoed.

RMNH 17651, 1 hgr.; RMNH 17657, 1 ㅇ. Loc.: "Avanavero road, km 219, Van Ams Creek, Nickerie District, Suriname". Leg.: M.S. Hoogmoed.

RMNH 17652, 1 hgr., 2 juv.; RMNH 17703, 1 juv. Loc.: "Blanche Marie cataracts, Nickerie River, Nickerie District, Suriname”. Leg.: M.S. Hoogmoed and D. Reeder.

RMNH 17653, 1 우. Loc.: "4 km SE Kraka, Brokopondo District, Suriname”. Leg.: M.S. Hoogmoed.

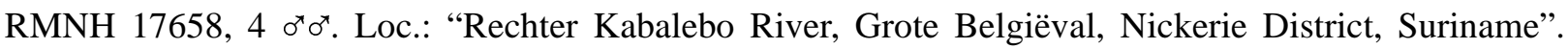
Leg.: M.S. Hoogmoed.

RMNH 17660, 1 hgr.; RMNH 17769, 1 hgr. Loc.: "Reynold's camp (Grassalco), Mozes Creek, Nickerie District, Suriname". Leg.: M.S. Hoogmoed and W.N. Polder.

RMNH 17661, 1 우 RMNH 17668, 2 hgr.; RMNH 17673, 1 hgr.; RMNH 17682, 6 hgr. Loc.: "Brownsberg, 450-500 m., Brokopondo District, Suriname”. Leg.: M.S. Hoogmoed, W.N. Polder.

RMNH 17662, 1 juv.; RMNH 17663, 1 o`, 8 우우, 4 hgr.; RMNH 17669, 1 hgr. Loc.: "Coppename River, Raleigh cataracts, Saramacca District, Suriname”. Leg.: C.A. Mees and M.S. Hoogmoed.

RMNH 17664, 2 우; RMNH 17670, 1 우 RMNH 17674, 1 우 RMNH 17679, 1 hgr.; RMNH 17680, 1

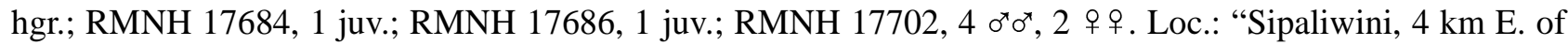
airstrip, Nickerie District, Suriname". Leg.: M.S. Hoogmoed.

RMNH 17665, 5 hgr. Loc.: "foot of Brownsberg, Brokopondo District, Suriname". Leg.: M.S. Hoogmoed. 
RMNH 17666, 1 hgr. Loc.: "Brownsberg, 2 km E of Suralco house, Brokopondo District, Suriname". Leg.: M.S. Hoogmoed.

RMNH 17671, 1 hgr. Loc.: "Brownsberg, near Suralco house, Brokopondo District, Suriname". Leg.: M.S. Hoogmoed.

RMNH 17672, 1 hgr. Loc.: "encampment Tigri, New River (upper Corantyne River), Nickerie District, Suriname”. Leg.: M.S. Hoogmoed.

RMNH 17678, 2 hgr. Loc.: "Sipaliwini, 2 km N Vier Gebroeders Mt., Nickerie District, Suriname”. Leg.: M.S. Hoogmoed.

RMNH 17683, 1 hgr. Loc.: "SE of airstrip, Kayser Mts., Nickerie District, Suriname”. Leg.: M.S. Hoogmoed.

RMNH 17685, 1 juv.; RMNH 17700, 1 ๙ . Loc.: "Sipaliwini, E. of airstrip, Nickerie District, Suriname". Leg.: M.S. Hoogmoed.

RMNH 17687, 1 hgr. Loc.: "Paloemeu airstrip, Marowijne District, Suriname”. Leg.: M.S. Hoogmoed.

RMNH 17688, 1 hgr. Loc.: “camp 8, Mapane Area, Commewijne District, Suriname”. Leg.: M.S. Hoogmoed.

RMNH 17689, 1 spec. Loc.: “upper Gran Rio, Brokopondo District, Suriname”. Leg.: J.F. Hulk.

RMNH 17690, 1 ㅇ, 1 hgr. Loc.: "Lucie River, Nickerie District, Suriname". Leg.: J.F. Hulk.

RMNH 17691, 7 spec. Loc.: "Guyana Gold Placer, Brokopondo District, Suriname”. Leg.: W.C. van Heurn.

RMNH 17692, 1 우 1 ơ. Loc.: "Hebiweri, Saramacca District, Suriname”. Leg.: D.C. Geijskes.

RMNH 17693, 2 우우, 1 hgr., 1 juv. Loc.: "Wilhelmina Mountains, transect I, Saramacca District, Suriname”. Leg.: D.C. Geijskes.

RMNH 17694, 1 hgr. Loc.: "Wilhelmina Mountains, transect II, Saramacca District, Suriname". Leg.: D.C. Geijskes.

RMNH 17695, 1 hgr. Loc.: "Rechter Coppename River, Saramacca District, Suriname". Leg.: D.C. Geijskes.

RMNH 17696, 1 hgr. Loc.: "trail Toekoemoetoe Creek to Tafelberg, km 3, Saramacca District, Suriname". Leg.: L. Schmidt.

RMNH 17697, 1 \%; RMNH 17698, 1 spec. Loc.: "railway km 121, foot of Browsberg, upper Mahami creek, Brokopondo District, Suriname”. Leg.: Suriname Expedition 1948-49.

RMNH 17699, 1 ㅇ. Loc.: "Nassau Mountains, km 1.1, Marowijne District, Suriname”. Leg.: Suriname Expedition 1948-49.

RMNH 17704, 1 ․ Loc.: "camp on Maratakka River, Nickerie District, Suriname”. Leg.: P. Staffeleu.

RMNH 17751, 1 juv. Loc.: "Rechter Kabalebo River, Brokobotoval, Nickerie District, Suriname”. Leg.:

S.B. Kroonenberg.

RMNH 17752, 1 spec. Loc.: "3 km S of Reynold's camp, Mozes Creek, Nickerie District, Suriname". Leg.: M.S. Hoogmoed and W.N. Polder.

RMNH 17753, 2 우우. Loc.: "Vreedzaam Creek, $10 \mathrm{~km}$ N of Lucie River, Nickerie District, Suriname". Leg.: M.S. Hoogmoed.

RMNH 17754, 2 우우 RMNH 17755, 1 ㅇ․ Loc.: "Eva Creek, 20 km N of Lucie River, Nickerie District, Suriname". Leg.: M.S. Hoogmoed.

RMNH 17756, 1 ㅇ. Loc.: "Rechter Kabalebo River, 10 km S K. vallen, Nickerie District, Suriname". Leg.: M.S. Hoogmoed.

RMNH 17757, 1 ㅇ. Loc.: "Rechter Kabalebo River, 2nd falls, Nickerie District, Suriname”. Leg.: M.S. Hoogmoed.

RMNH 17766, 1 o $^{\star}$ Loc.: "Lucie River, $10 \mathrm{~km}$ NE of Coeroeni airstrip, Nickerie District, Suriname". Leg.: M.S. Hoogmoed. 
RMNH 17767, 1 spec. Loc.: “10-20 km N Lucie River, Nickerie District, Suriname”. Leg.: M.S. Hoogmoed.

RMNH 17771, 1 ơ. Loc.: "Rechter Kabalebo River, K. vallen, Nickerie District, Suriname”. Leg.: M.S. Hoogmoed.

RMNH 17772, 1 juv. Loc.: “Rechter Kabalebo River, Doublestepsval, Nickerie District, Suriname”. Leg.: M.S. Hoogmoed.

RMNH 17773, 1 juv. Loc.: "Kabalebo River, Avanavero cataracts, Nickerie District, Suriname". Leg.: M.S. Hoogmoed and W.N. Polder.

RMNH 17776, $1 \sigma^{\star}$. Loc.: “Linker Kabalebo River, river camp, Nickerie District, Suriname”. Leg.: S.B. Kroonenberg.

RMNH 4900, 1 spec. Loc.: "Coppename River, Saramacca District, Suriname”. Leg.: H. van Capelle.

\section{Geotrypetes seraphini occidentalis Parker, 1936}

Parker, 1936. Zool. Meded. Leiden, 19: 99.

Syntypes: RMNH 6527, 1 ㅇ. Loc.: "Grand Cape Mount, Liberia”. Leg.: A.F. Demery.

RMNH 6528, 2 o $^{x}$, 1 ․ . Loc.: Liberia. Leg.: J. Demery.

Remarks. RMNH 6527 was identified as a specimen of Schistometopum gregorii (Boulenger, 1895) by E. Taylor. According to Taylor (1968: 725) syntypes are in the Muséum National d'Histoire Naturelle in Paris (MNHNP 20.188-189) and the Natural History Museum in London (BM 1930.11.19.50, 1935.9.2.67 [ex RMNH], BM 1935.9.2.68 [ex RMNH], 1934.6.6.8, 1907, 10.25.10, 1917.4.13.33-35 and 1927.9.27.164).

\section{Hyla (Litoria) arfakiana Peters \& Doria, 1878}

Peters \& Doria, 1878. Ann. Mus. Civ. Stor. Nat., 13: 421; tab. VI, fig. 2.

Current name: Litoria arfakiana (Peters \& Doria, 1878)

Paralectotypes: RMNH 4241, 4 spec. Loc.: "Hatam" [Arfak Mountains, Irian Jaya, Indonesia]. Leg.: O. Beccari. Received: 1875.

Remarks. The lectotype (MSNG 29723 A) was selected by Capocaccia (1957: 213) and is in the Museo Civico di Storia Naturale Giacomo Doria in Genova. The paralectotypes are in the Natural History Museum in London (BM 82.10.3.3-5), the Museum für Naturkunde der Humboldt-Universität zu Berlin (ZMB 9756, 3 spec.), the Museo Civico di Storia Naturale Giacomo Doria in Genova (MSNG 29723, 9 spec.) and the Royal Belgian Institute of Natural Sciences in Brussels (ex RMNH).

\section{Hyla amboinensis Horst, 1883}

Horst, 1883. Notes Leyden Mus., 5: 239.

Current name: Litoria amboinensis (Horst, 1883)

Syntypes: RMNH 4418, 1 ㅇ, 1 ơ. Loc.: “Amboina” [Ambon, Moluccas, Indonesia]. Leg.: D. Hoedt. Received: 1866.

RMNH 4419, 1 ơ. Loc.: “Misool” [Moluccas, Indonesia]. Leg.: D. Hoedt. Received: 1866.

\section{Hyla aruensis Horst, 1883}

Horst, 1883. Notes Leyden Mus., 5: 242.

Current name: Litoria aruensis (Horst, 1883) 
Syntypes: RMNH 4416A-B, 2 spec. Loc.: "Aroe-Islands" [Aru Island, Indonesia]. Leg.: C.B.H. von Rosenberg.

RMNH 4417, 1 spec. Loc.: "Misool” [Moluccas, Indonesia]. Leg.: D. Hoedt.

\section{Hyla aurifasciata Schlegel, 1837}

Schlegel, 1837-1844. Abbild. Neuer Unvollst. Amph., 1: 27; tab. IX, fig. 4.

Current name: Philautus aurifasciatus (Schlegel, 1837)

Lectotype: RMNH 4266 A, 1 spec. Loc.: "innern bergigen Gegenden der Insel Java” [Indonesia]. Leg.: S. Müller.

Paralectotypes: RMNH 4266 B-F, 5 spec. Loc.: "innern bergigen Gegenden der Insel Java” [Indonesia]. Leg.: S. Müller.

Remarks. RMNH 4266 A was designated lectotype by Bossuyt \& Dubois (2001: 12). Though the publication date on the first page of Schlegel's Abbildungen is 1837-1844, the work was issued in parts. See Stejneger's Herpetology of Japan (1907: 540) for the publication dates.

\section{Hyla Bernsteini Horst, 1883}

Horst, 1883. Notes Leyden Mus., 5: 241.

Current name: Litoria nigropunctata (Meyer, 1874).

Syntype: RMNH 4421, 1 spec. Loc.: "Gebeh" [Irian Jaya, Indonesia]. Leg.: H.A. Bernstein.

Remarks. According to Duellman (1977: 135) there is only one type specimen, whereas Horst (1883: 241) referred to "Two specimens from Salawattie and Gebeh collected by Bernstein". A second specimen from Salawatti could not be located in the Leiden musem.

\section{Hyla boesemani Goin, 1966}

Goin, 1966a. Zool. Meded. Leiden, 41: 229; fig. 1.

Current name: Scinax boesemani (Goin, 1966)

Holotype: RMNH 12601, 1 ๙ . Loc.: "near Zanderij, Suriname District, Suriname”. Leg.: C.J. Goin and D.C. Geijskes.

Paratypes: RMNH 12602-12603, 2 o $^{x} o^{x}$ Loc.: "near Zanderij, Suriname District, Suriname”. Leg.: C.J. Goin and D.C. Geijskes.

Remarks. Other paratypes are in the Florida State Museum (FSM 22367), the Coleman J. Goin Collection [now in the Museum of Northern Arizona in Flagstaff] (CJG 2408-09, 2414, 2385-87), the American Museum of Natural History in New York (A75555), the Natural History Museum in London (BM 1966.26), the Carnegie Museum in Pittsburgh (CM 41595), the Museum of Comparitive Zoology in Cambridge, Massachusetts (MCZ 52833) and the National Museum of Natural History in Washington (USNM 159140).

\section{Hyla bürgeri Temminck \& Schlegel, 1838}

Temminck \& Schlegel, 1838. Fauna Japonica, 3: 113; pl. 3, figs. 7, 8.

Current name: Buergeria buergeri (Temminck \& Schlegel, 1838)

Syntypes: RMNH 1692, 2 spec. Loc.: “Japon”. Leg.: H. Bürger.

\section{Hyla capitula Tyler, 1968}

Tyler, 1968. Zool. Verh. Leiden, 96: 64; pl. I, b; fig. 19.

Current name: Litoria capitula (Tyler, 1968) 
Holotype: RMNH 5317, 1 gravid ㅇ․ Loc.: "Samlakki, Tenimbar Island" [Saumlaki, Maluku, Indonesia]. Leg.: F. Kopstein.

\section{Hyla chalconotus Schlegel, 1837}

Schlegel, 1837-1844. Abbild. Neuer Unvollst. Amph., 1: 23; pl. IX, fig. 1.

Current name: Hydrophylax chalconotus (Schlegel, 1837)

Syntypes: RMNH 5364, 1 spec. Loc.: "Java" [Indonesia]. Leg.: S. Müller. possibly RMNH 4264, 4 spec. Loc.: "Java" [Indonesia]. Leg.: H. Boie and H.C. Macklot.

Remarks. The type localities are "Java" (figured specimen in Schlegel, 1837: plate IX) and "Sumatra" (Schlegel, 1837: 25). S. Müller collected specimens of $R$. chalconota from both localities, but the specimens from Sumatra could not be located in the Leiden museum. The specimens from RMNH 4264 are not explicitly mentioned by Schlegel, but they are likely to have been examined, according to M.S. Hoogmoed (Leiden Museum records). Though the publication date on the first page of Schlegel's Abbildungen is 1837-1844, the work was issued in parts. See Stejneger's Herpetology of Japan (1907: 540) for the publication dates.

\section{Hyla epacrorhina Duellman, 1972}

Duellman, 1972. Zool. Meded. Leiden, 47: 183; pl. I, fig. I.

Current name: Scinax garbei (Miranda-Ribeiro, 1926).

Paratype: RMNH 16922 (formerly KU 139248), 1 spec. Loc.: "Pilcopata, Departamento Cuzco, Perú, $13^{\circ} 51^{\prime}$ 'S, $71^{\circ} 12^{\prime} W^{\prime}$ '. Leg.: W.E. Duellman and T.H. Fritts.

Remarks. Other paratypes are in the Museum of Natural History, University of Kansas in Lawrence (holotype: KU 139247; paratypes: 139242-45) and in the American Museum of Natural History in New York (AMNH 85382).

\section{Hyla erythraea Schlegel, 1837}

Schlegel, 1837-1844. Abbild. Neuer Unvollst. Amph., 1: 27; pl. IX, fig. 3.

Current name: Hylarana erythraea (Schlegel, 1837)

Syntypes: RMNH 1744, 4 spec. Loc.: "Sumatra” [Indonesia]. Leg.: S. Müller.

RMNH 1746, 9 spec. Loc.: "Sumatra" [Indonesia]. Leg.: S. Müller.

RMNH 1749, 1 spec. Loc.: "Sumatra" [Indonesia]. Leg.: S. Müller.

RMNH 4265, 1 spec. Loc.: "Java” [Indonesia]. Leg.: S. Müller.

Remarks. Other syntypes are in the Muséum National d'Histoire Naturelle in Paris (MNHNP 4570-4572).

Though the publication date on the first page of Schlegel's Abbildungen is 1837-1844, the work was issued in parts. See Stejneger's Herpetology of Japan (1907: 540) for the publication dates.

\section{Hyla genimaculata Horst, 1883}

Horst, 1883. Notes Leyden Mus., 5: 240.

Current name: Litoria genimaculata (Horst, 1883)

Holotype: RMNH 4420, 1 spec. Loc.: “Gebeh (Gagie?)” [Indonesia]. Leg.: H.A. Bernstein.

\section{Hyla Horstockii Schlegel, 1837}

Schlegel, 1837-1844. Abbild. Neuer Unvollst. Amph., 1: 24.

Current name: Hyperolius horstockii (Schlegel, 1837) 
Lectotype: RMNH 1775, 1 spec. Loc.: "Vorgebirge der guten Hoffnung" [Cape of Good Hope, South Africa]. Leg.: H.B. van Horstok.

Paralectotypes: RMNH 10770, 2 spec. Loc.: "Vorgebirge der guten Hoffnung" [Cape of Good Hope, South Africa]. Leg.: H.B. van Horstok.

Remarks. The lectotype was designated by Poynton (1963: 332). Though the publication date on the first page of Schlegel's Abbildungen is 1837-1844, the work was issued in parts. See Stejneger's Herpetology of Japan (1907: 540) for the publication dates.

\section{Hyla leporosa Schlegel, 1844}

Schlegel, 1837-1844. Abbild. Neuer Unvollst. Amph., 1: 105.

Current name: Theloderma leporosum Tschudi, 1838

Syntype: RMNH 1754, 1 ex. Loc.: "Padang auf Sumatra" [Indonesia]. Leg.: S. Müller.

Remarks. This specimen is also syntype of Theloderma leporosa Tschudi, 1838. Though the publication date on the first page of Schlegel's Abbildungen is 1837-1844, the work was issued in parts. See Stejneger's Herpetology of Japan (1907: 540) for the exact dates of publication. Frost (2006) however gives 1837 as publication date for all new species described by Schlegel in his Abbildungen.

\section{Hyla margaritifera Schlegel, 1844}

Schlegel, 1837-1844. Abbild. Neuer Unvollst. Amph., 1: 107.

Current name: Rhacophorus margaritifer (Schlegel, 1844)

Syntypes: RMNH 1911, 2 spec. Loc.: "Java" [Indonesia]. Leg.: H. Kuhl and J.C. van Hasselt.

Remarks. Though the publication date on the first page of Schlegel's Abbildungen is 1837-1844, the work was issued in parts. See Stejneger's Herpetology of Japan (1907: 540) for the publication dates. Frost (2006) however gives 1837 as publication date for all new species described by Schlegel in his Abbildungen.

\section{Hyla mystax Van Kampen, 1906}

Van Kampen, 1906. Nova Guinea, 5: 173, fig. 6.

Current name: Litoria mystax (Van Kampen, 1906)

Holotype: RMNH 4632, 1 ơ. Loc.: "Moaif” [Irian Jaya, Indonesia]. Leg.: Nieuw Guinea Expeditie, 1903.

\section{Hyla nigerrima Spix, 1824}

Spix, 1824. Nova Spec. Nov. Test. Ran. Brasil: 36, pl. IX, fig. 2.

Current name: Ameerega trivittata (Spix, 1824)

Lectotype: RMNH 1799, 1 ơ. Loc.: “juxta pagum Ecgá” [Ega, Tefé, Brazil]. Leg.: J.B. von Spix.

Remarks. Hoogmoed \& Gruber (1983: 368) considered RMNH 1799 to be the "only 'surviving' syntype" and selected it as lectotype of Hyla nigerrima Spix, 1824.

\section{Hyla Reinwardtii Schlegel, 1844}

Schlegel, 1837-1844. Abbild. Neuer Unvollst. Amph., 1: 105; tab. XXX, figs. 1-4.

Current name: Rhacophorus reinwardtii (Schlegel, 1844)

Syntypes: RMNH 1870, 2 spec. Loc.: Java, Indonesia. Leg.: H. Boie and H.C. Macklot. RMNH 6517, 2 spec. Loc.: Java, Indonesia. Leg.: H. Boie and H.C. Macklot. 
RMNH 3899, 1 spec. Loc.: Sumatra, Indonesia. Leg.: S. Müller.

Remarks. The type locality given by Schlegel (1844: 106) is "Java"and "Sumatra". Though the publication date on the first page of Schlegel's Abbildungen is 1837-1844, the work was issued in parts. See Stejneger's Herpetology of Japan (1907: 540) for the publication dates. Frost (2006) however gives 1837 as publication date for all new species described by Schlegel in his Abbildungen.

\section{Hyla rubra Laurenti, 1768}

Laurenti, 1768. Spec. Med. Exhib. Synops. Rept.: 5.

Current name: Scinax ruber (Laurenti, 1768).

Neotype: RMNH 25883 (formerly RMNH 15922 B), 1 ơ. Loc.: Paramaribo Botanical Garden, Paramaribo, Suriname. Leg.: H.W.C. Cossee.

Remarks. The neotype (from Paramaribo, Surinam) was designated by Duellman \& Wiens (1993: 37).

\section{Hyla trivittata Spix, 1824}

Spix, 1824. Nova Spec. Nov. Test. Ran. Brasil: 35; pl. IX, fig. 1.

Current name: Ameerega trivittata (Spix, 1824)

Lectotype: RMNH 1836, 1 ơ. Loc.: "juxta flumen Teffé” [Tefé, Brazil]. Leg.: J.B. von Spix.

Remarks. The lectotype was selected by Hoogmoed \& Gruber (1983: 368). The paralectotype (ZSMH 42/

$0)$ is in the Zoologisches Staatssammlung München in Munich.

\section{Hyla variabilis Boulenger, 1896}

Boulenger, 1896. Ann. Mag. Nat. Hist., Ser. 6, 17: 20.

Current name: Dendropsophus columbianus (Boettger, 1892)

Syntype: RMNH 4496, 1 spec. Loc.: "Cali” [Colombia]. Leg.: W.F.H. Rosenberg.

Remarks. Other syntypes are in the Natural History Museum in London (BM 1947.2.13.15-31), the Naturhistorisches Museum Wien in Vienna (NHMW 19438.1-3), the Museo di Zoologia Universitá di Torino (MZUT An337), the Field Museum of Natural History in Chicago (FMNH 3565), the Museum of Comparative Zoology in Cambridge, Massachusetts (MCZ 2606), the Museum of Zoology, University of Michigan (UMMZ 46464, 51269, 58908), the National Museum of Natural History in Washington (USNM 71115) and the Zoologisches Museum in Hamburg (ZMH A00963).

\section{Hyla variolosa Spix, 1824}

Spix, 1824. Nova Spec. Nov. Test. Ran. Brasil: 37; pl. IX, fig.4.

Current name: Hypsiboas punctatus (Schneider, 1799)

Lectotype: RMNH 1879, 1 ㅇ. Loc.: Brasil. Leg.: J.B. von Spix.

Remarks. The lectotype was selected by Hoogmoed \& Gruber (1983: 366), who considered it the last remaining specimen of the type series. The type locality given by Spix (1824: 37) is "fluminis Amazonum" [Rio Amazonas]; given as “Amazonian Brazil” by Hoogmoed \& Gruber (1983: 366).

\section{Hyla wisselensis Tyler, 1968}

Tyler, 1968. Zool. Verh. Leiden, 96: 180; pl. 4, b-d; fig. 58.

Current name: Litoria wisselensis (Tyler, 1968) 
Holotype: RMNH 12295, 1 gravid 우 Loc.: "Enarotali, Lake Paniai, Wissel Lakes, Snow Mountains, New Guinea" [Irian Jaya, Indonesia]. Leg.: M. Boeseman and L.B. Holthuis.

Paratypes: RMNH 12296-12310, 15 spec. Loc.: "Enarotali, Lake Paniai, Wissel Lakes, Snow Mountains, New Guinea" [Irian Jaya, Indonesia]. Leg.: M. Boeseman and L.B. Holthuis.

Remarks. Five paratypes are in the South Australian Museum in Adelaide (SAMA 5539-5543).

\section{Hylaplesia borbonica Tschudi, 1838}

Tschudi, 1838. Classif. Batr.: 70.

Current name: Leptophryne borbonica (Tschudi, 1838)

Syntypes: RMNH 1739, 2 spec. Loc.: "India orientalis" [Indonesia]. Leg.: H. Boie and H.C. Macklot.

Remarks. The type locality given by Tschudi (1838: 70) is "India orientalis", restricted to "Java" by Inger (1966: 75, as Cacophryne borbonica).

\section{Hylella Cappellei Van Lidth de Jeude, 1904}

Van Lidth de Jeude, 1904. Notes Leyden Mus., 25: 94.

Current name: Hyalinobatrachium fleischmanni (Boettger, 1893)

Holotype: RMNH 4463, 1 spec. Loc.: "river Saramacca and its neighbourhood" [Suriname]. Leg.: Saramacca Expeditie, 1903.

\section{Hylodes grandoculis Van Lidth de Jeude, 1904}

Van Lidth de Jeude, 1904. Notes Leyden Mus., 25: 93.

Current name: Eleutherodactylus marmoratus (Boulenger, 1900)

Holotype: RMNH 4467, 1 spec. Loc.: "the basin and the sources of the Coppename" [Suriname] Leg.: Coppename Expeditie, 1901.

\section{Hylophorbus amboinensis Mertens, 1930}

Mertens, 1930. Zool. Meded. Leiden, 13: 145; fig. 1.

Current name: Callulops fuscus (Peters, 1867)

Holotype: RMNH 5315, 1 o. Loc.: “Ambon, Molukken” [Indonesia]. Leg.: F. Kopstein.

\section{Hylophorbus kopsteini Mertens, 1930}

Mertens, 1930. Zool. Meded. Leiden, 13: 147; fig. 2.

Current name: Callulops kopsteini (Mertens, 1930)

Holotype: RMNH 5310 A, 1 ๙ . Loc.: "Sanana, Soela-Inseln” [Sulawesi, Indonesia]. Leg.: F. Kopstein.

Paratypes: RMNH 5310 B-C, 1 o`, 1 ㅇ․ Loc.: "Sanana, Soela-Inseln" [Sulawesi, Indonesia]. Leg.: F. Kopstein.

\section{Hyperolius flavoviridis Peters, 1854}

Peters, 1854. Ber. Bekanntmach. Geeignet. Verhandl. Königl. Preuss. Akad. Wiss. Berlin, 1854: 628.

Current name: Hyperolius argus Peters, 1854 
Syntypes: possibly RMNH 1780, 1 spec. Loc.: Tete, Mozambique. Leg.: W.C.H. Peters.

Possibly RMNH 1785, 1 spec. Loc.: Tete, Mozambique. Leg.: W.C.H. Peters.

Remarks. Bauer et al. (1995: 44) indicated RMNH 1780 as a possible syntype. Syntypes are also in the Museum für Naturkunde der Humboldt-Universität zu Berlin (ZMB 6631-6632).

\section{Hyperolius milnei Loveridge, 1935}

Loveridge, 1935. Bull. Mus. Comp. Zool., 79: 18.

Current name: Hyperolius pusillus (Cope, 1862)

Paratypes: RMNH 6435, 2 spec. Loc.: Mombosasa, "Witu, Coast Province, Kenia Colony". Leg.: A. Loveridge. Ex: Museum of Comparative Zoology in Cambridge, Massachusetts.

Remarks. The holotype (MCZ 20025) is in the Museum of Comparative Zoology in Cambridge, Massachusetts.

\section{Ichthyophis asplenius Taylor, 1965}

Taylor, 1965. Kansas Univ. Sci. Bull., 46: 278; figs. 14, 15.

Current name: Caudacaecilia asplenia (Taylor, 1965)

Holotype: RMNH 11483 (formerly RMNH 6912 B), 1 spec. Loc.: "Boven Mahakkam, Borneo" [Indonesia]. Leg.: A.W. Nieuwenhuis.

Paratype: RMNH 11482 (formerly RMNH 6912 A), 1 spec. Loc.: "Boven Mahakkam, Borneo" [Indonesia]. Leg.: A.W. Nieuwenhuis.

Remarks. The holotype was formerly numbered RMNH 6912 B, under which it was listed by Taylor (1965: 278). RMNH 11482 was also renumberd from RMNH 6912 A.

\section{Ichthyophis atricollaris Taylor, 1965}

Taylor, 1965. Kansas Univ. Sci. Bull., 46: 267; figs. 5, 6.

Holotype: RMNH 10684, 1 spec. Loc.: "Long Bloee, Boven Mahakkam, Borneo" [Indonesia]. Leg.: A.W. Nieuwenhuis.

Paratypes: RMNH 6912 D, 1 spec. Loc.: "Boven Mahakkam, Borneo" [Indonesia]. Leg.: A.W. Nieuwenhuis.

RMNH 10685, 1 spec. Loc.: Balik Papan, Borneo. Leg.: Lampmeinert.

Remarks. The locality given by Taylor (1965: 267) for RMNH 10685 ("Long Bloee, Boven Mahakkam, Borneo") is not consistent with the locality on the jar label nor with the RMNH records. According to these, the locality for RMNH 10685 is "Balik Papan, Borneo" [Balikpapan, Kalimantan Timur, Indonesia].

\section{Kalophrynus pleurostigma Tschudi, 1838}

Tschudi, 1838. Classif. Batr.: 86.

Holotype: RMNH 2279, 1 spec. Loc.: "Sumatra" [Indonesia]. Leg.: S. Müller.

Remarks. This specimen is in very poor condition, it is dried out and therefore fragile.

\section{Lechriodus aganoposis Zweifel, 1972}

Zweifel, 1972b. Am. Mus. Novit., 2507: 9.

Paratypes: RMNH 17005, 1 spec. Loc.: "West Mt. Antares, 20 km W Sibil (Mabilibol), West Irian" [Indonesia]. Leg.: Nederlandse Nieuw Guinea Expeditie, 1959. 
RMNH 17006 A-D, 4 spec. Loc.: "Sibil, Star Mountains, West Irian" [Indonesia]. Leg.: Nederlandse Nieuw Guinea Expeditie, 1959.

RMNH 17007, 1 spec. Loc.: "probably Katem, 20 km S. and 20 km E. Sibil, Star Mountains, West Irian" [Indonesia]. Leg.: Nederlandse Nieuw Guinea Expeditie, 1959.

Remarks. The holotype (AMNH 75791) is in the American Museum of Natural History in New York.

\section{Lechriodus platyceps Parker, 1940}

Parker, 1940. Novit. Zool., 42: 28.

Paratype: RMNH 4234, 1 ơ. Loc.: "Haddam (Hatam), Arfak. Mts" [Irian Jaya, Indonesia]. Leg.: O. Beccari. Remarks. The holotype is in the Natural History Museum in London (BM 76.7.18.6) from "Arfak, Dutch New Guinea".

\section{Leptobrachium Hasseltii Tschudi, 1838}

Tschudi, 1838. Classif. Batr:: 81.

Current name: Leptobrachium hasseltii Tschudi, 1838.

Lectotype: RMNH 2015, 1 q. Loc.: "Java" [Indonesia]. Leg.: H. Boie and H.C. Macklot. Paralectotype: RMNH 2014, 1 ․ Loc.: "Java" [Indonesia]. Leg.: H. Boie and H.C. Macklot. Remarks. RMNH 2015 was designed lectotype by Dubois \& Ohler (1998: 16).

\section{Megalobatrachus Sieboldii Tschudi, 1837}

Tschudi, 1837, Neues Jahrb. Mineral. Geogn. Geol. Petrefactenkunde, 5: 547.

Current name: Andrias japonicus (Temminck, 1836)

Lectotype: RMNH 2392, 1 juv. Loc.: “Japan”. Leg.: Ph.F. von Siebold.

Paralectotypes: RMNH 2394, 1 spec. Loc.: "Japan”. Leg.: Ph.F. von Siebold.

RMNH 18562, 1 ad. skeleton. Loc.: "Japan".

Remarks. The lectotype was selected by Hoogmoed (1978: 102). There is also a paralectotype in the Muséum National d'Histoire Naturelle in Paris (MNHNP 7614). These specimens are also the type series of Salamandra maxima Temminck \& Schlegel, 1838 and of Triton japonicus Temminck, 1836.

\section{Megophrys montana Kuhl \& Van Hasselt, 1822}

Kuhl \& Van Hasselt, 1822b. Algemeene Konst- en Letter-Bode, 7: 102.

Syntypes: RMNH 2212, 4 spec. Loc.: "Java” [Indonesia]. Leg.: H. Kuhl and J.C. van Hasselt.

Remarks. Kuhl \& Van Hasselt described this species in a later publication (1822a: 475) under the name Megophrys monticola, an incorrect original spelling of Megophrys montana according to the International Commission on Zoological Nomenclature (ICZN, 1994: 84).

\section{Metopostira macra Van Kampen, 1906}

Van Kampen, 1906. Nova Guinea, 5: 167; figs. 1, 2.

Current name: Hylophorbus rufescens Macleay, 1878

Holotype: RMNH 4631, 1 spec. Loc.: “Am Moso und Tami” [Mosso River, Papua New Guinea]. Leg.: Nieuw Guinea Expeditie, 1903.

\section{Microcaecilia taylori Nussbaum \& Hoogmoed, 1979}

Nussbaum \& Hoogmoed, 1979. Zool. Meded. Leiden, 54: 225; pl. I.

Holotype: RMNH 15165 A, 1 ơ. Loc.: "Vier Gebroeders Mnt., Sipaliwini, Surinam”. Leg.: M.S. Hoogmoed. 
Paratypes: RMNH 15165 B, 1 ๙`. Loc.: "Vier Gebroeders Mnt., Sipaliwini, Surinam”. Leg.: M.S. Hoogmoed.

RMNH 17832, 1 ㅇ․ Loc.: "rainforest surrounding savanna, Sipaliwini, Surinam”. Leg.: M.S. Hoogmoed and J.J.P. Paats.

\section{Microhyla achatina Tschudi, 1838}

Tschudi, 1838. Classif. Batr.: 28, 71.

Syntypes: RMNH 1728, 5 spec. Loc.: "Java” [Indonesia]. Leg.: S. Müller and H.C. Macklot.

Remarks. There are also syntypes in the Bokermann Collection (now in the Museu de Zoologia, Universidade de São Paulo), the Natural History Museum in London (possibly including BM 44.2.22.97) and the Institue Royal des Sciences Naturelles de Belgique in Brussels (IRSNB 1.722), all obtained through exchange with the National Museum of Natural History in Leiden.

\section{Myobatrachus paradoxus Schlegel, 1850}

Schlegel, 1850. Proc. Zool. Soc. London: 9.

Current name: Myobatrachus gouldii (Gray, 1841)

Holotype: RMNH 2060, 1 spec. Loc.: S. O. de l'Australie [Western Australia]. Leg: Dr. Preiss. Remarks. The type locality given by Schlegel (1850: 9) is “Australia; Swan River".

\section{Ololygon danae Duellman, 1986}

Duellman, 1986. Copeia, 1986: 864, fig. 1.

Current name: Scinax danae (Duellman, 1986)

Paratypes: RMNH 21966-21967, 1 ơ, 1 ㅇ. Loc.: "from km 127 on El Dorado-Santa Elena de Uairén road, Estado de Bolívar, Venezuela, 1250 m. (0557’N, 61²7’W)”. Leg.: W.E. Duellman and J.E. Simmons.

Remarks. Other types are in the Museum of Natural History, University of Kansas in Lawrence (holotype: KU 167073; paratopotypes: KU 167070, 167072, 167074-83, 167088-91) and in the Natural History Museum in London (BM 1979.562-63).

\section{Ololygon exigua Duellman, 1986}

Duellman, 1986. Copeia: 866, fig. 3.

Current name: Scinax exiguus (Duellman, 1986)

Paratypes: RMNH 21964-21965, 2 o $^{*}$. Loc.: "from km 144 on El Dorado-Santa Elena de Uairén road in the Gran Sabana, Estado de Bolívar, Venezuela, 1210 m. (0553’N, 61²3’W)”. Leg.: W.E. Duellman, J.R. León, J.E. Simmons and L. Trueb.

Remarks. Other types are in the Museum of Natural History, University of Kansas in Lawrence (holotype: KU 167094; paratypes: KU 167093, 167095-125, 167130, 16792) and in the Natural History Museum in London (BM 1979.564-64).

\section{Ololygon trilineata Hoogmoed \& Gorzula, 1979}

Hoogmoed \& Gorzula, 1979. Zool. Meded. Leiden, 54: 193; pl. I, figs. a-d.

Current name: Scinax trilineatus (Hoogmoed \& Gorzula, 1979)

Holotype: RMNH 18257, 1 오. Loc.: "12 km SE. El Manteco, Estado Bolívar, Venezuela”. Leg.: M.S. Hoogmoed and S.J. Gorzula. 


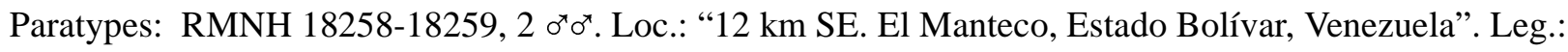
M.S. Hoogmoed and S.J. Gorzula.

RMNH 18260, 1 hgr. Loc.: "base of Vier Gebroeders Mountain, Sipaliwini, Distr. Nickerie, Suriname". Leg.: M.S. Hoogmoed.

Remarks. Other paratypes are in the American Museum of Natural History in New York (AMNH 43637, 46248, 97944 - 97949).

\section{Onychodactylus Schlegeli Tschudi, 1838}

Tschudi, 1838. Classif. Batr.: 92.

Onychodactylus gen. nov. Tschudi, 1838. Classif. Batr.: 92.

Current name: Onychodactylus japonicus (Houttuyn, 1782)

Lectotype: RMNH 2292 A, 1 ơ. Loc.: "Japan”. Leg.: Ph.F. von Siebold.

Paralectotypes: RMNH 2292 B-D, 1 ox, 2 우오. Loc.: "Japan”. Leg.: Ph.F. von Siebold.

RMNH 2289, 20 dried ad. Loc.: "Japan". Leg.: Ph.F. von Siebold.

RMNH 2290, 5 우 우, 3 hgr. Loc.: "Japan”. Leg.: H. Bürger.

RMNH 2291, 7 larvae. Loc.: “Japan”. Leg.: Ph.F. von Siebold.

RMNH 18560, 1 skeleton. Loc.: "Japan".

Remarks. Hoogmoed (1978: 97) designated the lectotype. There are also paralectotypes in the Natural History Museum in London and in the Muséum National d'Histoire Naturelle in Paris (MNHNP 4692), obtained through exchange with the National Museum of Natural History in Leiden. RMNH 2289 consists of 20 dried specimens with a large hole in the head as they were used for pharmaceutical purposes. These specimens are also the type series of Salamandra unguiculata Temminck \& Schlegel, 1838.

\section{Ooidozyga semipalmata Smith, 1927}

Smith, 1927. Proc. Zool. Soc. London, 1927: 203; pl. II, fig. 3.

Current name: Occidozyga semipalmata Smith, 1927

Paratypes: RMNH 6888 (formerly M. Smith 8740), 1 spec. Loc.: "Lowah, near Mt. Bonthain” [S. Sulawesi, Indonesia]. Leg.: M.A. Smith.

RMNH 6889 (formerly M. Smith 8546), 1 spec. Loc.: Djikoro, Mt. Bonthaim [S. Sulawesi, Indonesia]. Leg.: M.A. Smith.

Remarks. The holotype is in the Natural History Museum in London (M. Smith 8728).

\section{Oreophryne idenburgensis Zweifel, 1956}

Zweifel, 1956. Am. Mus. Novit, 1766: 27.

Paratype: RMNH 10473 (formerly AMNH 49571), 1 spec. Loc.: "18 km. Southwest of Bernhard Camp, Idenburg River, Netherlands New Guinea" [Irian Jaya, Indonesia]. Leg.: W.B. Richardson. Ex: American Museum of Natural History in New York.

Remarks. The holotype (AMNH A49663) and paratypes (AMNH 4966-69) are in the American Museum of Natural History in New York.

\section{Oxyglossus lima Tschudi, 1838}

Tschudi, 1838. Classif. Batr.: 85.

Current name: Occidozyga lima (Gravenhorst, 1829)

Syntypes: RMNH 2247-2247a, 14 spec. Loc.: "Java”. Leg.: H. Boie and H.C. Macklot. 
Remarks. Tschudi (1838: 85) examined specimens from "Mus. Lugd. Par. et Francof." [the National Museum of Natural History in Leiden, the Muséum National d'Histoire Naturelle in Paris and the Forschungsinstitut und Natur-Museum Senckenberg in Frankfurt].

\section{Pelophryne rhopophilius Inger \& Stuebing, 1996}

Inger \& Stuebing, 1996. Raffles Bull. Zool., 44: 547; fig. 3.

Paratype: RMNH 14555, 1 spec. Loc.: "Mt. Damus (1200 m), Sambas, West Kalimantan” [Indonesia]. Leg.: H. Hallier.

Remarks. The holotype and paratypes are in the Field Museum of Natural History in Chicago (FMNH 252422, and FMNH 252423-24, respectively).

\section{Philautus similis Van Kampen, 1923}

Van Kampen, 1923. Amph. Indo-Austral. Arch.: 273.

Holotype: RMNH 5066, 1 spec. Loc.: "Sumatra (Mt. Talamau, 1200 m.)” [Indonesia]. Leg.: E. Jacobson.

\section{Phractops alutaceus Peters, 1867}

Peters, 1867. Monatsber. Preuss. Akad. Wiss. Berlin: 31.

Current name: Litoria novaehollandiae (Steindachner, 1867)

Paratype: possibly RMNH 3867, 1 spec. Loc.: “Rockhampton, O. Australien” [Queensland, Australia]. Ex: Museum Godeffroy. Received: 1877.

Remarks. The holotype is probably in the Museum für Naturkunde der Humboldt-Universität zu Berlin (ZMB 5653), and possible paratypes are in the National Museum of Natural History in Leiden (RMNH 3867) and in the American Museum of Natural History in New York (AMNH 23581). Bauer et al. (1995: 43) suggested that the RMNH specimen was likely not part of the type series. However, in accordance with the Leiden museum records ("Rockhampton, O. Australië, Goddeff.", received in 1877) RMNH 3867 could have been one of the specimens described by Peters, provided that this specimen was part of the first shipment to the Museum Godeffroy from their collector in Rockhampton, in 1867.

\section{Phrynobatrachus alleni Parker, 1936}

Parker, 1936. Zool. Meded. Leiden, 19: 91.

Paratypes: RMNH 6594, 20 spec. Loc.: "Soforeh Place, Liberia”. Leg.: J. Büttikofer and J.A. Sala.

Remarks. The holotype is in the Natural History Museum in London (BM 1947.2.4.33, formerly BM 1929.6.1.12). Paratypes are also in London (BM 1927.9.27.112-117; BM 1935.9.2.14-19) and in the Museum of Comparative Zoology in Cambridge, Massachusetts (MCZ 11946-7, 11961-70, 11951-60).

\section{Phrynobatrachus brongersmai Parker, 1936}

Parker, 1936. Zool. Meded. Leiden, 19: 90.

Current name: Phrynobatrachus ogoensis (Boulenger, 1906)

Holotype: RMNH 6591, 1 ơ. Loc.: "Grand Cape Mount" [Liberia]. Leg.: A.F. Demery.

Paratypes: RMNH 6592, 1 ‥ Loc.: "Soforeh Place" [Liberia]. Leg.: J. Büttikofer and J.A. Sala.

RMNH 6593, 1 ơ. Loc.: "Liberia". Leg.: J. Demery.

Remarks. Other paratypes are in the Natural History Museum in London (BM 1935.9.2.20, BM 1935.9.2.21). 


\section{Phrynomantis glandulosa Zweifel, 1972}

Zweifel, 1972a. Bull. Am. Mus. Nat. Hist., 148: 469; fig. 29.

Current name: Callulops glandulosus (Zweifel, 1972)

Holotype: RMNH 16667, 1 ơ. Loc.: "Mt. Kerewa, 3340 m. (10.960 ft.), Southern Highlands District, Territory of Papua" [Papua New Guinea]. Leg.: W. Vink.

\section{Phrynomantis infulata Zweifel, 1972}

Zweifel, 1972a. Bull. Am. Mus. Nat. Hist., 148: 476; figs. 34, 35.

Current name: Mantophryne infulata (Zweifel, 1972)

Paratype: RMNH 24225 (formerly AMNH 66686), 1 spec. Loc.: "Arau, Kratke Mountains, elevation 4600 ft. (1400 m.), Eastern Highlands District, Territory of New Guinea” [Papua New Guinea]. Leg.: Archbold Expedition. Ex: American Museum of Natural History in New York.

Remarks. The holotype (AMNH 66687) and paratypes (AMNH 66684-85, 66688-66703) are in the American Museum of Natural History in New York.

\section{Phrynopus lucida Cannatella, 1984}

Cannatella, 1984. Occas. Pap. Mus. Nat. Hist. Univ. Kansas, 113: 6.

Current name: Eleutherodactylus lucida (Cannatella, 1984)

Paratypes: RMNH 17822 (formerly WED 47190-91), 2 spec. Loc.: "N. slope Abra Tapuna, Ayacucho, Peru". Leg.: W.E. Duellman and J.E. Simmons. Ex: University of Kansas in Lawrence.

Remarks. The holotype (KU 162435) as well as most of the paratypes (KU 162427-34, 162436-45, 162446-59) are at the Museum of Natural History, University of Kansas in Lawrence. There are also paratypes in the Field Museum of Natural History in Chicago (FMNH 204170-76).

\section{Physalaemus aguirrei Bokermann, 1966}

Physalaemus aguirrei Bokermann, 1966b. Physis (Buenos Aires), 26 : 194.

Paratype: RMNH 13901 (formerly WCAB 19309), 1 spec. Loc.: "Refugio Sooretama, Linhares, Espírito Santo, Brasil". Leg.: F.M. Oliveira and W.C.A. Bokermann. Ex: Bokermann Collection.

Remarks. The holotype is in the Bokermann Collection (WCAB 19303, now Museu de Zoologia, Universidade de São Paulo).

\section{Physalaemus obtectus Bokermann, 1966}

Physalaemus obtectus Bokermann, 1966b: 197.

Paratype: RMNH 14052 (formerly WCAB 20620), 1 spec. Loc.: "Refugio Sooretama, Linhares, Espírito Santo, Brasil". Leg.: F.M. Oliveira and W.C.A. Bokermann. Ex: Bokermann Collection.

Remarks. The holotype is in the Bokermann Collection (WCAB 20498, now Museu de Zoologia, Universidade de São Paulo).

\section{Pleurodeles waltl Michahelles, 1830}

Michahelles, 1830. Isis v. Oken, 1830: 195.

Lectotype: RMNH 2379, 1 ad. ‥ Loc.: Espagne. Leg.: C.W. Michahelles.

Remarks. The lectotype was designated by Hoogmoed (1978: 103). The type locality given by Michahelles (1830: 191) is "Chiclana in Südspanien" and "Hispania meridionali" (column 195), restricted to "Chi- 
clana, southern Spain" by Hoogmoed (1978: 103). This specimen is also the holotype of Salamandra pleurodeles Temminck \& Schlegel, 1838.

\section{Pleurodema Bibroni Tschudi, 1838}

Tschudi, 1838. Classif. Batr.: 47, 85 .

Current name: Pleurodema bibroni Tschudi, 1838

Paralectotype: possibly RMNH 2277, 1 spec. Loc.: Valparaiso [Chile]. Leg.: C. Gaudichaud.

Remarks. According to Frost (1985: 255) this is the specimen Tschudi (1838: 85) referred to as "Syn. Bombinator ocellatus Mus. Lugd.”. It actually belongs to Pleurodema thaul (Lesson, 1827). The data on the label does not match the data of the original publication (Tschudi, 1838: 48): "Hr. d'Orbigny brachte es in mehrern Exemplaren von Monte-Video nach Paris". Cei (1962: 92) gave "Chile (Penco, Concepción)" as type locality. Ortiz and Lescure (1989: 118) designated MNHNP 4501 (in the Muséum National d'Histoire Naturelle, Paris) as lectotype.

\section{Polypedates Junghunii Bleeker, 1856}

Bleeker, 1856. Natuurkd. Tijdschr. Nederl. Indie, 11: 469.

Current name: Hydrophylax chalconotus (Schlegel, 1837)

Syntype: RMNH 3962, 1 spec. Loc.: Indische Archipel. Ex.: Bleeker Collection. Received: 1880.

Remarks. Bleeker (1856: 470) mentioned several specimens collected by F.W. Junghuhn: "de heer Junghuhn ondekte deze soort bij het meer Patengan op Java" [Mr. Junghun discovered this species near lake Patengan in Java]. According to the Auction Catalogue of the Bleeker Collection (1879: 59), Bleeker kept in his collection only one specimen of this species, now RMNH 3962.

\section{Pseudobufo subasper Tschudi, 1838}

Tschudi, 1838. Classif. Batr.: 87.

Syntypes: RMNH 2199, 1 spec. Loc.: "Borneo" [Indonesia]. Leg.: S. Müller.

RMNH 2200, 1 spec. Loc.: "Borneo" [Indonesia].

RMNH 2216, 1 spec. Loc.: "Borneo" [Indonesia].

\section{Pyxicephalus adspersus Tschudi, 1838}

Tschudi, 1838. Classif. Batr.: 84.

Syntypes: RMNH 2258, 2 spec. Loc.: "Promontorium Bonæ Spei" [Cape of Good Hope, South Africa]. Leg.: P.A. Delalande.

Remarks. Other syntypes are in the Muséum National d'Histoire Naturelle in Paris (MNHNP 4525 and 4527).

\section{Pyxicephalus Delalandii Tschudi, 1838}

Tschudi, 1838. Classif. Batr.: 46, 84.

Current name: Tomopterna delalandii (Tschudi, 1838)

Syntypes: RMNH 2260, 1 spec. Loc.: "Promontorium Bonæ Spei” [Cape of Good Hope, South Africa]. Leg.: P.A. Delalande. 
RMNH 2261, 2 spec. Loc.: "Promontorium Bonæ Spei" [Cape of Good Hope, South Africa]. Leg.: H.B. van Horstok.

Remarks. Other syntypes are in the Muséum National d'Histoire Naturelle in Paris (MNHNP 721, 4521, and 4529).

\section{Pyxicephalus edulis Peters, 1854}

Peters, 1854. Ber. Bekanntmach. Geeignet. Verhandl. Königl. Preuss. Akad. Wiss. Berlin, 1854: 626.

Syntype: RMNH 2274, 1 spec. Loc.: Mozambique. Leg.: W.H.C. Peters.

Remarks. Other syntypes are in the Museum für Naturkunde der Humboldt Universität zu Berlin (ZMB $3349,3350,10056-58,50260,50290$, and 50301-02). According to Boulenger (1882: 34), a syntype may be a specimen in the Natural History Museum in London, from "Mossambique", received from W. Peters. The type locality ("Mossambique, Boror, Tette") was restricted to "Tete" by Loveridge (1953: 375).

\section{Rana aurea Lesson, 1827}

Lesson, 1827. In Duperrey (ed.), Voyage Coquille, Zool., 2: 60; pl. 7; fig. 2.

Current name: Litoria aurea (Lesson, 1827)

Paralectotype: possibly RMNH 1814, 1 spec. Loc.: Blauwe bergen, Nieuw Holland [Blue Mountains, New South Wales, Australia]. Don.: R.P. Lesson.

Remarks. Roux-Estève (1979: 26) designated MNHNP 4857 from Port Jackson as lectotype. The lectotype and a paralectotype (MNHNP 537) are in the Muséum National d'Histoire Naturelle in Paris. However, in accordance with the type locality given in the original publication ("la rivière Macquarie a Bathurst, au-delà des montagnes Bleues"), RMNH 1814 was probably also part of the type series described by Lesson.

\section{Rana ibanorum Inger, 1964}

Inger, 1964. Fieldiana, Zool., $44: 151$.

Current name: Limnonectes ibanorum (Inger, 1964)

Paratype: RMNH 14534, 1 spec. Loc.: "Long Petah, Kalimantan” [Indonesia]. Leg.: H.C. Siebers.

Remarks. The holotype (FMNH 76894) and paratypes are in the Field Museum of Natural History in Chicago.

\section{Rana Kuhlii Tschudi, 1838}

Tschudi, 1838. Classif. Batr.: 40.

Current name: Limnonectes kuhlii (Tschudi, 1838)

Syntypes: RMNH 4297, 2 spec. Loc.: "Java" [Indonesia].

Remarks. There are also syntypes in the Muséum Nationale d'Histoire Naturelle in Paris (MNHNP 4469).

\section{Rana limnocharis Gravenhorst, 1829}

Gravenhorst, 1829. Delic. Mus. Zool. Vratislav., 1: 42.

Current name: Fejervarya limnocharis (Gravenhorst, 1829)

Neotype: RMNH 4287, 1 ․ Loc.: Java, Indonesia. Leg.: H. Kuhl and J.C. van Hasselt.

Remarks. The neotype was designated by Dubois and Ohler (2000: 28). They restricted the locality to “near Buitenzorg [now Bogor] (06 35'S, 106 47’E), West Java, Java, Indonesia”. RMNH 4287 is also the neotype of Rana limnocharis Wiegmann, 1834. 


\section{Rana limnocharis Wiegmann, 1834}

Wiegmann, 1834. Nova Acta Phys. Med. Acad. Caesar Leopold Carol., 17: 225.

Current name: Fejervarya limnocharis (Gravenhorst, 1829)

Neotype: RMNH 4287, 1 ․ Loc.: Java, Indonesia. Leg.: H. Kuhl and J.C. van Hasselt.

Remarks. The neotype was designated by Dubois and Ohler (2000: 28, 36). They also restricted the locality to “near Buitenzorg [now Bogor] (06 35'S, 106 47'E), West Java, Java, Indonesia”. RMNH 4287 is also the neotype of Rana limnocharis Gravenhorst, 1829.

\section{Rana macularia var. javanica Horst, 1883}

Horst, 1883. Notes Leyden Mus., 5: 243.

Current name: Sylvirana nicobariensis (Stoliczka, 1870)

Syntypes: RMNH 14022, 15 spec. Loc.: "Java” [Indonesia]. Leg.: S. Müller.

\section{Rana paramacrodon kenepaiensis Inger, 1966}

Inger, 1966. Fieldiana, Zool., 52: 232.

Current name: Limnonectes kenepaiensis (Inger, 1966)

Holotype: RMNH 14519, 1 ơ. Loc.: “Merkata, Kenepai Mountains, western Kalimantan” [Indonesia]. Leg.: J. Büttikoffer, Borneo Expedition, 1894.

Remarks. There is also a paratype in the Field Museum of Natural History in Chicago (FMNH 121306).

\section{Rana pentadactyla Laurenti, 1768}

Laurenti, 1768. Spec. Med. Exhib. Synops. Rept.: 32.

Current name: Leptodactylus pentadactylus (Laurenti, 1768)

Neotype: RMNH 29559, 1 o`. Loc.: Suralcokamp V, Lelygebergte, distr. Marowijne, Suriname. Leg.: M.S. Hoogmoed.

Remarks. Heyer (2005: 310) designated the neotype. The type locality of Rana pentadactyla Laurenti, 1768 is "Indiis", restricted to "Suriname" by Müller (1927: 276).

\section{Rana pygmaea Spix, 1824}

Spix, 1824. Nova Spec. Nov. Test. Ran. Brasil: 30; pl. 6, fig. 2.

Current name: Leptodactylus ocellatus (Linnaeus, 1758)

Paratype: possibly RMNH 2041, 1 spec. Loc.: Brésil.

Remarks. According to Heyer (1973: 26) the holotype from the Zoologisches Staatssammlung München is now lost. Hoogmoed \& Grüber (1983: 356) indicated that type material may have never been in the Zoologisches Staatssammlung and that RMNH 2041 probably belongs to the type series, but they "refrain from considering it a syntype".

\section{Rana rugosa Temminck \& Schlegel, 1838}

Temminck \& Schlegel, 1838. Fauna Japonica, 3: 110; pl. 3, figs. 3, 4.

Current name: Glandirana rugosa (Temminck \& Schlegel, 1838)

Syntypes: RMNH 2064, 9 spec. Loc.: "Japon”. Leg.: Ph.F. von Siebold.

Remarks. Stejnejer (1907: 123) considered the type locality to be "probably Nagasaki". 


\section{Ranoidea Jacksoniensis Tschudi, 1838}

Tschudi, 1838. Classif. Batr.: 35, 76.

Current name: Litoria aurea (Lesson, 1831)

Paralectotypes: possibly RMNH 1721, 1 spec. Loc.: Port Jackson [Australia]. Leg.: F. Péron and C.A. Lesuer. Possibly RMNH 1722, 1 spec. Loc.: N.Z. Wales [New South Wales, Australia]. Don.: Verreaux.

Remarks. Tschudi (1838) mentioned specimens "von Perón auf Neu-Holland gefunden" (p. 35) and from "Nova Hollandia" (p. 76), in the "Mus. Lugd. et Par." (the National Museum of Natural History in Leiden and the Muséum National d'Histoire Naturelle in Paris). Other types are possibly MNHNP 537 and MNHNP 4858, the latter regarded as lectotype by Guibé (1950: 21).

\section{Rhacophorus bifasciatus Van Kampen, 1923}

Van Kampen, 1923. Amph. Indo-Austral. Arch.: 257.

Syntypes: RMNH 6519 A-B, 2 spec. Loc.: Soengei Koembang, Korintji, Sumatra, Indonesia. Leg.: E. Jacobson.

RMNH 5062 (5 spec.), RMNH 5063 (2 spec.) Loc.: Soengei Koembang, Korintji, Sumatra, Indonesia. Leg.: E. Jacobson.

Remarks. The two specimens in RMNH 6519 A-B belong to Rhacophorus poecilonotus Boulenger, 1920. The type locality given by Van Kampen is "Sumatra (Sungai Kring, Sungai Kumbang, 1600m., and Sinlak Deras in Kurintji)". Both specimens from RMNH 5063 are in very poor condition.

\section{Salamandra Genei Temminck \& Schlegel, 1838}

Temminck \& Schlegel, 1838. Fauna Japonica, 3: 115.

Current name: Hydromantes genei (Temminck \& Schlegel, 1838)

Lectotype: RMNH 2296 A, 1 ox. Loc.: "Sardaigne" [Sardinia, Italy]. Leg.: F.J. Cantraine.

Paralectotypes: RMNH 2296 B-G, 3 우우, 3 hgr. Loc.: "Sardaigne" [Sardinia, Italy]. Leg.: F.J. Cantraine and C.G. Gené.

Remarks. Hoogmoed (1978: 103) designated the lectotype.

\section{Salamandra maxima Temminck \& Schlegel, 1838}

Temminck \& Schlegel, 1838. Fauna Japonica, 3: 127; pls. VI-VIII.

Current name: Andrias japonicus (Temminck, 1836)

Lectotype: RMNH 2392, 1 juv. Loc.: Japon. Leg.: Ph.F. von Siebold.

Paralectotypes: RMNH 2394, 1 spec. Loc.: Japon. Leg.: Ph.F. von Siebold.

RMNH 18562, 1 ad. skeleton. Loc.: Japon.

Remarks. The type locality given by Temminck \& Schlegel (1838: 135) is "Sakanosta,.., des monts Suzuga jama" and "mont Okude". The lectotype was selected by Hoogmoed (1978: 102). There is a paralectotype in the Muséum National d'Histoire Naturelle in Paris (MNHNP 7614), and although not included in Hoogmoed (1978), Bauer et al. (1993: 290) listed ZMB 30646 as paralectotype, in the Museum für Naturkunde der Humboldt-Universität zu Berlin, obtained through exchange with the Leiden museum. All these specimens are also the type series of Megalobatrachus Sieboldii Tschudi, 1838 and of Triton japonicus Temminck, 1836. 


\section{Salamandra naevia Temminck \& Schlegel, 1838}

Temminck \& Schlegel, 1838. Fauna Japonica, 3: 122; pl. IV, figs. 4-6; pl. V, figs. 9-10.

Current name: Hynobius naevius (Temminck \& Schlegel, 1838)

Lectotype: RMNH 2306 A, 1 ad. Loc.: "Japon”. Leg.: Ph.F. von Siebold.

Paralectotypes: RMNH 2306 B-K, 7 ad., 3 hgr. Loc.: "Japon”. Leg.: Ph.F. von Siebold.

RMNH 2305, 2 larvae. Loc.: "Japon”. Leg.: Ph.F. von Siebold.

RMNH 18559, 1 ad. skeleton. Loc.: "Japon".

Remarks. Hoogmoed (1978: 96) designated RMNH 2306 A as lectotype and considered the type locality as "dans les contrées montueuses des îles Nippon et Sikok, particulièrement dans les provinces Sagami, Sinano, Tanba, Tazima et Tosa, situées entre les 33 et 36 degrés de latitude boreale" [Honshu and Shikoku Islands, Japan]. Hoogmoed listed paralectotypes in the Naturhistorisch Museum Wien in Vienna (NHMW 2290), the Museum of Comparative Zoology in Cambridge, Massachusetts (MCZ 7365), the Natural History Museum in London, the Muséum National d'Histoire Naturelle in Paris (MNHNP 4690) and the Museum für Naturkunde der Humboldt-Universität zu Berlin (ZMB 3619, 2 ad.), obtained through exchange with the National Museum of Natural History in Leiden. Bauer et al. (1993: 292) observed that there is no evidence of exchange for this material between the Leiden en the Vienna museums. In that case, the status of NHMW 2290 should be considered doubtful.

\section{Salamandra nebulosa Temminck \& Schlegel, 1838}

Temminck \& Schlegel, 1838. Fauna Japonica, 3: 127; pl. IV, figs. 7-9.

Current name: Hynobius nebulosus (Temminck \& Schlegel, 1838)

Lectotype: RMNH 2309 A, 1 ㅇ. Loc.: Japon. Leg.: Ph.F. von Siebold.

Paralectotypes: RMNH 2309 B-T, 9 o $^{x}, 3$ hgr. ${o^{x}}^{x}, 5$ 우우, 2 juv. Loc.: Japon. Leg.: Ph.F. von Siebold.

RMNH 2307, 1 larva. Loc.: "Japon”. Leg.: H. Bürger.

Remarks. The type locality given by Temminck \& Schlegel (1838: 127) for Von Siebold's specimens is "monts Mits jama (les trois monts) situés dans les environs de Nagasaki" [Japan]. Hoogmoed (1978: 100) designated RMNH 2309 A, an adult female, as lectotype. There are also paralectotypes in the Natural History Museum in London, the Forschungsinstitut und Natur-Museum Senckenberg in Frankfurt, the Museum für Naturkunde der Humboldt-Universität zu Berlin (ZMB 3620, 2 ad.) and the Muséum National d'Histoire Naturelle in Paris (MNHNP 4691, 4691 A).

\section{Salamandra pleurodeles Temminck \& Schlegel, 1838}

Temminck \& Schlegel, 1838. Fauna Japonica, 3: 117.

Current name: Pleurodeles waltl Michahelles, 1830

Holotype: RMNH 2379, 1 ad. ㅇ․ Loc.: "Espagne méridionale". Leg.: C.W. Michahelles.

Remarks. Hoogmoed (1978: 103) gives as type locality "southern Spain". This specimen is also the lectotype of Pleurodeles waltl Michahelles, 1830.

\section{Salamandra scutata Temminck \& Schlegel, 1838}

Temminck \& Schlegel, 1838. Fauna Japonica, 3: 119.

Current name: Hemidactylium scutatum (Temminck \& Schlegel, 1838)

Holotype: RMNH 2301, 1 spec. Loc.: "Nashville au Tennessee” [U.S.A.]. Leg.: G. Troost. 


\section{Salamandra subcristata Temminck \& Schlegel, 1838}

Temminck \& Schlegel, 1838. Fauna Japonica, 3: 125; pl. IV, figs. 1-3; pl. V, figs. 7-8.

Current name: Cynops pyrrhogaster (Boie, 1826)

Lectotype: RMNH 2397 A, 1 ․ Loc.: "Japon”. Leg.: H. Bürger.

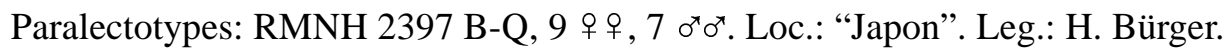

RMNH 18561, 1 ad. skeleton. Loc.: "Japon".

Remarks. Hoogmoed (1978: 98) designated the lectotype and restricted the type locality to "Nagasaki". Other paralectotypes are in the Muséum National d'Histoire Naturelle in Paris (MNHNP 4711, 4711A) and in the Museum of Comparative Zoology in Cambridge, Massachusetts (MCZ 21327).

\section{Salamandra unguiculata Temminck \& Schlegel, 1838}

Temminck \& Schlegel, 1838. Fauna Japonica, 3: 123; pl. V, figs. 1-6.

Current name: Onychodactylus japonicus (Houttuyn, 1782)

Lectotype: RMNH 2292 A, 1 ox. Loc.: Japon. Leg.: Ph.F. von Siebold.

Paralectotypes: RMNH 2292 B-D, $10^{x}, 2$ 우오. Loc.: Japon. Leg.: Ph.F. von Siebold.

RMNH 2289, 20 dried ad. Loc.: Japon. Leg.: Ph.F. von Siebold.

RMNH 2290, 5 우 우 3 hgr. Loc.: Japon. Leg.: H. Bürger.

RMNH 2291, 7 larvae. Loc.: Japon. Leg.: Ph.F. von Siebold.

RMNH 18560, 1 skeleton. Loc.: Japon.

Remarks. The type locality given by Temminck \& Schlegel (1838: 125) is "îles Nippon et Sikok, particulièrement dans les provinces Sagamai, Sinano, Tanba, Tazima et Tosa, situées entre les 33 et 36 degrés de latitude boreale" [Honshu and Shikoku Islands, Japan]. Hoogmoed (1978: 97) designated the lectotype. There are also paralectotypes in the Natural History Museum in London, the Muséum National d'Histoire Naturelle in Paris (MNHNP 4692), the Museum of Comparative Zoology in Cambridge, Massachusetts (MCZ 21320) and the Museum für Naturkunde der Humboldt-Universität zu Berlin (ZMB 3633, larva), obtained through exchange with the National Museum of Natural History in Leiden. These specimens are also the type series of Onychodactylus Schlegeli Tschudi, 1838.

\section{Stefania riae Duellman \& Hoogmoed, 1984}

Duellman \& Hoogmoed, 1984. Misc. Publ. Mus. Nat. Hist. Univ. Kansas, 75: 23; fig. 21.

Paratype: RMNH 31974 (formerly KU 17489), 1 ơ. Loc.: "Cerro Sarisariñama, 1400 m, Estado Bolívar, Venezuela”. Leg.: P. Bermudes.

Remarks. Other types are in the Museum of Natural History, University of Kansas in Lawrence (KU 174688, holotype) and the National Museum of Natural History in Washington (USMN 212331, paratype).

\section{Theloderma leporosa Tschudi, 1838}

Tschudi, 1838. Classif. Batr.: 32, 74.

Current name: Theloderma leporosum Tschudi, 1838

Syntype: RMNH 1754, 1 spec. Loc.: "Sumatra" [Indonesia]. Leg.: S. Müller.

Remarks. Tschudi (1838: 74) mentioned two specimens collected by S. Müller and deposited in Leiden ("Adult. et pull."). The second specimen is probably lost. This specimen is also syntype of Hyla leporosa Schlegel, 1844. 


\section{Trachycephalus atlas Bokermann, 1966}

Bokermann, 1966c. Neotropica, 12: 120.

Paratypes: RMNH 14050-14051 (formerly WCAB 33678-33679), 2 spec. Loc.: "Fazenda Santo Onofre, 10 km al este de Maracás, Bahía, Brasil”. Leg.: F.M. Oliveira \& W.C.A. Bokermann. Ex: Bokermann Collection.

Remarks. The holotype is in the Bokermann Collection (WCAB 33669, now in the Museu de Zoologia Universidade de São Paulo).

\section{Triton japonicus Temminck, 1836}

Temminck, 1836. Coup-d'oeil sur Faun. Îles Sonde et Emp. Jap.: xxvi.

Current name: Andrias japonicus (Temminck, 1836)

Lectotype: RMNH 2392, 1 juv. Loc.: "Japon”. Leg.: Ph.F. von Siebold.

Paralectotypes: RMNH 2394, 1 spec. Loc.: "Japon”. Leg.: Ph.F. von Siebold.

RMNH 18562, 1 ad. skeleton. Loc.: "Japon".

Remarks. The lectotype was selected by Hoogmoed (1978: 102). There are also paralectotypes in the Muséum National d'Histoire Naturelle in Paris (MNHNP 7614) and in the Museum für Naturkunde der Humboldt-Universität zu Berlin (ZMB 30646). These specimens are also the type series of Salamandra maxima Temminck \& Schlegel, 1838 and of Megalobatrachus Sieboldii Tschudi, 1838.

\section{Xenobatrachus anorbis Blum \& Menzies, 1989}

Blum \& Menzies, 1989. Alytes, 7: 140; fig. 8.

Current name: Xenorhina anorbis (Blum \& Menzies, 1989)

Paratype: RMNH 23513 (formerly included in RMNH 16619), 1 spec. Loc.: "Tenmasigin, Star Mountains, altitude 1900 m." [Irian Jaya, Indonesia]. Leg.: Nieuw Guinea Expeditie, 1959.

Remarks. The holotype is in the University of Papua New Guinea, Biology Department in Port Moresby (UP 5621). There are also paratypes in the National Museum and Art Gallery in Port Moresby (PM 2354a-c).

\section{Xenopus Boiei Wagler, 1827}

Wagler, 1827. Isis v. Oken, 20: 726.

Current name: Xenopus laevis (Daudin, 1802)

Syntypes: possibly RMNH 2267, 4 spec. Loc.: Cap [Cape of Good Hope, Rep. South Africa]. Leg.: H.B. van Horstok.

Remarks. The type material consisted of specimens from H. Boie's collection. Wagler (1827: 726) mentioned a specimen "im Leydner Cabinete" [RMNH]. The Leiden museum records and the oldest label on the jar indicate that the specimens from RMNH 2267 were first identified as Xenopus Boiei, suggesting that they belonged to the original type series.

\section{Xenorhina parkerorum Zweifel, 1972}

Zweifel, 1972a. Bull. Am. Mus. Nat. Hist., 148: 539; figs. 77, 78.

Paratypes: RMNH 16619, 1 spec. Loc.: “Tenmasigin, 6 km. N Mabilabol, West Irian” [Indonesia]. Leg.: Nieuw Guinea Expeditie, 1959.

RMNH 16666, 1 spec. Loc.: "Tibigi, Huria River, 1600 m (5250ft.), Southern Highlands District, Territory of Papua” [Papua New Guinea]. Leg.: W. Vink. 
Remarks. The holotype is in the Museum of Comparative Zoology in Cambridge, Massachusetts (MCZ 81678). Zweifel (1972a: 539) also listed paratypes in the American Museum of Natural History in New York (AMNH 84589, 84555), in the Museum of Comparitive Zoology in Cambridge, Massachusetts (MCZ 80509) and in the South African Museum in Cape Town (SAM 6404, 6405). 


\section{Appendix:}

Alfabetical list of the current names for the type specimens in the RMNH

Adelophryne adiastola Hoogmoed \& Lescure, 1984

Adelophryne baturitensis Hoogmoed, Borges \& Cascon, 1994

Adelophryne maranguapensis Hoogmoed, Borges \& Cascon, 1994

Ameerega trivittata (Spix, 1824) [Hyla nigerrima Spix, 1824; Hyla trivittata Spix, 1824]

Andinophryne colomai Hoogmoed, 1985

Andrias japonicus (Temminck, 1836) [Triton japonicus Temminck, 1836; Megalobatrachus Sieboldii Tschudi, 1837;

Salamandra maxima Temminck \& Schlegel, 1838]

Ansonia latidisca Inger, 1966

Ansonia leptopus (Günther, 1872) [Ansonia longidigita gryllivoca Inger, 1960]

Asterophrys turpicola (Schlegel, 1837) [Ceratophrys turpicola Schlegel, 1837]

Atelopus flavescens Duméril \& Bibron, 1841

Atelopus spumarius Cope, 1871 [Atelopus spumarius barbotini Lescure, 1981]

Aubria occidentalis Perret, 1994

Austrochaperina basipalmata (Van Kampen, 1906) [Chaperina basipalmata Van Kampen, 1906]

Austrochaperina macrorhyncha (Van Kampen, 1906) [Chaperina macrorhyncha Van Kampen, 1906]

Batrachylodes elegans Brown \& Parker, 1970

Boulengerula taitana (Loveridge, 1935) [Boulengerula taitanus Loveridge, 1935]

Buergeria buergeri (Temminck \& Schlegel, 1838) [Hyla bürgeri Temminck \& Schlegel, 1838]

Bufo asper Gravenhorst, 1829

Bufo japonicus Temminck \& Schlegel, 1838 [Bufo vulgaris var. japonicus Temminck \& Schlegel, 1838]

Bufo mauritanicus Schlegel, 1841

Callulops fuscus (Peters, 1867) [Hylophorbus amboinensis Mertens, 1930]

Callulops glandulosus (Zweifel, 1972) [Phrynomantis glandulosa Zweifel, 1972]

Callulops kopsteini (Mertens, 1930) [Hylophorbus kopsteini Mertens, 1930]

Caudacaecilia asplenia (Taylor, 1965) [Ichthyophis asplenius Taylor, 1965]

Chaunus granulosus (Spix, 1824) [Bufo granulosus barbouri Gallardo, 1965]

Chaunus ictericus (Spix, 1824) [Bufo ictericus Spix, 1824]

Chaunus marinus (Linnaeus, 1758) [Bufo albicans Spix, 1824]

Chaunus ornatus (Spix, 1824) [Bufo dorsalis Spix, 1824; Bufo ornatus Spix, 1824; Bufo Spixii Fitzinger, 1826]

Cochranella geijskesi (Goin, 1966) [Centrolenella geijskesi Goin, 1966]

Chioglossa lusitanica longipes Arntzen \& Alexandrino, in press

Cynops pyrrhogaster (Boie, 1826) [Salamandra subcristata Temminck \& Schlegel, 1838]

Dendrobates azureus Hoogmoed, 1969

Dendropsophus columbianus Boettger, 1892 [Hyla variabilis Boulenger, 1896]

Duttaphrynus melanostictus (Schneider, 1799) [Bufo gymnauchen Bleeker, 1858]

Eleutherodactylus chiastonotus Lynch \& Hoogmoed, 1977

Eleutherodactylus lucida (Cannatella, 1984) [Phrynopus lucida Cannatella, 1984]

Eleutherodactylus lynchi Duellman \& Simmons, 1977

Eleutherodactylus marmoratus (Boulenger, 1900) [Hylodes grandoculis Van Lidth de Jeude, 1904]

Eleutherodactylus zeuctotylus Lynch \& Hoogmoed, 1977

Euhyas alticola (Lynn, 1937) [Eleutherodactylus alticola Lynn, 1937]

Euhyas pinarensis (Dunn, 1926) [Eleutherodactylus pinarensis Dunn, 1926]

Fejervarya limnocharis (Gravenhorst, 1829) [Rana limnocharis Gravenhorst, 1829; Rana limnocharis Wiegmann, 1834]

Geotrypetes seraphini occidentalis Parker, 1936

Glandirana rugosa (Temminck \& Schlegel, 1838) [Rana rugosa Temminck \& Schlegel, 1838] 
Hemidactylium scutatum (Temminck \& Schlegel, 1838) [Salamandra scutata Temminck \& Schlegel, 1838]

Hyalinobatrachium fleischmanni (Boettger, 1893) [Hylella Cappellei Van Lidth de Jeude, 1904]

Hydromantes genei (Temminck \& Schlegel, 1838) [Salamandra Genei Temminck \& Schlegel, 1838]

Hydrophylax chalconotus (Schlegel, 1837) [Hyla chalconotus Schlegel, 1837; Polypedates Junghuhnii Bleeker, 1856]

Hylarana erythraea (Schlegel, 1837) [Hyla erythraea Schlegel, 1837]

Hylophorbus rufescens Macleay, 1878 [Metopostira macra Van Kampen, 1906]

Hyloxalus toachi (Coloma, 1995) [Colostethus toachi Coloma, 1995]

Hynobius naevius (Temminck \& Schlegel, 1838) [Salamandra naevia Temminck \& Schlegel, 1838]

Hynobius nebulosus (Temminck \& Schlegel, 1838) [Salamandra nebulosa Temminck \& Schlegel, 1838]

Hyperolius argus Peters, 1854 [Hyperolius flavoviridis Peters, 1854]

Hyperolius horstockii (Schlegel, 1837) [Hyla Horstockii Schlegel, 1837]

Hyperolius pusillus (Cope, 1862) [Hyperolius milnei Loveridge, 1935]

Hypsiboas punctatus (Schneider, 1799) [Hyla variolosa Spix, 1824]

Ichthyophis atricollaris Taylor, 1965

Ichthyophis hypocyaneus (Boie, 1827) [Coecilia hypocyanea Boie, 1827]

Kalophrynus pleurostigma Tschudi, 1838

Kaloula baleata (Müller in Oort \& Müller, 1833) [Bombinator baleatus Müller, 1833 in Oort \& Müller]

Lechriodus aganoposis Zweifel, 1972

Lechriodus platyceps Parker, 1940

Leptobrachium hasseltii Tschudi, 1838 [Leptobrachium Hasseltii Tschudi, 1838]

Leptodactylus ocellatus (Linnaeus, 1758) [Rana pygmaea Spix, 1824]

Leptodactylus pentadactlylus (Laurenti, 1768) [Rana pentadactyla Laurenti, 1768]

Leptophryne borbonica (Tschudi, 1838) [Hylaplesia borbonica Tschudi, 1838]

Leptophryne cruentata (Tschudi, 1838) [Bufo cruentatus Tschudi, 1838]

Limnonectes ibanorum (Inger, 1964) [Rana ibanorum Inger, 1964]

Limnonectes kenepaiensis (Inger, 1966) [Rana paramacrodon kenepaiensis Inger, 1966]

Limnonectes kuhli (Tschudi, 1838) [Rana Kuhlii Tschudi, 1838]

Litoria amboinensis (Horst, 1883) [Hyla amboinensis Horst, 1883]

Litoria arfakiana (Peters \& Doria, 1878) [Hyla (Litoria) arfakiana Peters \& Doria, 1878]

Litoria aruensis (Horst, 1883) [Hyla aruensis Horst, 1883]

Litoria aurea (Lesson, 1827) [Rana aurea Lesson, 1827; Ranoidea Jacksoniensis Tschudi, 1838]

Litoria capitula (Tyler, 1968) [Hyla capitula Tyler, 1968]

Litoria genimaculata (Horst, 1883) [Hyla genimaculata Horst, 1883]

Litoria inermis (Peters, 1867) [Chiroleptes inermis Peters, 1867]

Litoria mystax (Van Kampen, 1906) [Hyla mystax Van Kampen, 1906]

Litoria nigropunctata (Meyer, 1874) [Hyla Bernsteini Horst, 1883]

Litoria novaehollandiae (Steindachner, 1867) [Phractops alutaceus Peters, 1867]

Litoria wisselensis (Tyler, 1968) [Hyla wisselensis Tyler, 1968]

Mantophryne infulata (Zweifel, 1972) [Phrynomantis infulata Zweifel, 1972]

Megophrys montana Kuhl \& van Hasselt, 1822

Megophrys nasuta (Schlegel, 1858) [Ceratophryne nasuta Schlegel, 1858]

Microcaecilia taylori Nussbaum \& Hoogmoed, 1979

Microhyla achatina Tschudi, 1838

Myobatrachus gouldii (Gray, 1841) [Myobatrachus paradoxus Schlegel, 1850]

Occidozyga lima (Gravenhorst, 1829) [Oxyglossus lima Tschudi, 1838]

Occidozyga semipalmatus (Smith, 1927) [Oxidozyga semipalmata Smith, 1927]

Onychodactylus japonicus (Houttuyn, 1782) [Onychodactylus Schlegeli Tschudi, 1838; Salamandra unguiculata Temminck \& Schlegel, 1838]

Oreophryne frontifasciata (Horst, 1883) [Callula frontifasciata Horst, 1883] 
Oreophryne idenburgensis Zweifel, 1956

Pelophryne rhopophilius Inger \& Stuebing, 1996

Pelorius nortoni Schwartz, 1976 [Cornufer unicolor Tschudi, 1838]

Philautus aurifasciatus (Schlegel, 1837) [Hyla aurifasciata Schlegel, 1837]

Philautus similis Van Kampen, 1923

Phrynobatrachus alleni Parker, 1936

Phrynobatrachus ogoensis (Boulenger, 1906) [Phrynobatrachus brongersmai Parker, 1936]

Phrynoidis juxtaspera (Inger, 1964) [Bufo juxtasper Inger, 1964]

Physalaemus aguirrei Bokermann, 1966

Physalaemus albifrons (Spix, 1824) [Bufo albifrons Spix, 1824]

Physalaemus obtectus Bokermann, 1966

Pleurodeles walt Michahelles, 1830 [Pleurodeles walt Michahelles, 1830; Salamandra pleurodeles Temminck \& Schlegel, 1838]

Pleurodema bibroni Tschudi, 1838 [Pleurodema Bibroni Tschudi, 1838]

Proceratophrys boiei (Wied-Neuwied, 1824) [Ceratophrys Boiei Wied-Neuwied, 1824]

Pseudepidalea brongersmai (Hoogmoed, 1972) [Bufo brongersmai Hoogmoed, 1972]

Pseudobufo subasper Tschudi, 1838

Pyxicephalus adpersus Tschudi, 1838

Pyxicephalus edulis Peters, 1854

Rhacophorus bifasciatus Van Kampen, 1923

Rhacophorus margaritifer (Schlegel, 1844) [Hyla margaritifera Schlegel, 1844]

Rhacophorus reinwardtii (Schlegel, 1837) [Hyla Reinwardtii Schlegel, 1837]

Scinax boesemani (Goin, 1966) [Hyla boesemani Goin, 1966]

Scinax danae (Duellman, 1986) [Ololygon danae Duellman, 1986]

Scinax exiguus (Duellman, 1986) [Ololygon exigua Duellman, 1986]

Scinax garbei (Miranda-Ribeiro, 1926) [Hyla epacrorhina Duellman, 1972]

Scinax ruber (Laurenti, 1768) [Hyla rubra Laurenti, 1768]

Scinax trilineatus (Hoogmoed \& Gorzula, 1979) [Ololygon trilineata Hoogmoed \& Gorzula, 1979]

Siphonops annulatus (Mikan, 1820) [Caecilia annulata Wagler, 1824]

Stefania riae Duellman \& Hoogmoed, 1984

Sylvirana nicobariensis (Stoliczka, 1870) [Rana macularia var. javanica Horst, 1883]

Tachycnemis seychellensis (Duméril \& Bibron, 1841) [Eucnemis seychellensis Tschudi, 1838]

Theloderma leporosum Tschudi, 1838 [Theloderma leporosa Tschudi, 1838; Hyla leporosa Schlegel, 1844]

Tomopterna delalandii (Tschudi, 1838) [Pyxicephalus Delalandii Tschudi, 1838]

Trachycephalus atlas Bokermann, 1966

Uraeotyphlus oxyurus (Duméril \& Bibron, 1841) [Caecilia oxyura Duméril \& Bibron, 1841]

Xenopus laevis (Daudin, 1802) [Xenopus boiei Wagler, 1827]

Xenorhina anorbis (Blum \& Menzies, 1989) [Xenobatrachus anorbis Blum \& Menzies, 1989]

Xenorhina oxycephala (Schlegel, 1858) [Bombinator oxycephalus Schlegel, 1858]

Xenorhina parkerorum Zweifel, 1972

Xenorhina similis (Zweifel, 1956) [Asterophrys similis Zweifel, 1956]

\section{References}

Anonymous (1879) Catalogue des Collections formées et laissées par M. P. Bleeker. dont la vente se fera par le ministère de Mr. J.M.E. Dercksen notaire, Lundi 1 Décembre 1879. à Leide (Pays-Bas). De Breuk \& Smits, Leiden, 71 pp.

Arntzen, J.W., Groenenberg, D.S.J., Alexandrino, J., Sequeira, F., Ferrand, N. (in press). Geographical variation in the Golden-striped salamander, Chioglossa lusitanica Bocage, 1864 and the description of a newly recognized subspecies. Journal of Natural History. 
Bauer, A.M., Günther, R., Klipfel, M. (1995) The herpetological contributions of Wilhelm C.H. Peters (1815-1883). 714 pp.

Bauer, A.M., Good, D.A, Günther, R. (1993) An Annotated Type Catalogue of the Caecilians and Salamanders (Amphibia: Gymnophiona and Caudata) in the Zoolohical Museum, Berlin. Mitteilungen aus dem Zoologischen Museum in Berlin, 69, 285-306.

Bleeker, P. (1856) Bestuursvergadering gehouden den 11n juni 1856, ten huize van den heer Steenstra Toussaint. Natuurkundig tijdschrift voor Nederlandsch-Indië, 11, 465-470.

Bleeker, P. (1858) Bestuursvergadering gehouden den 12n november 1857, ten huize van den heer Steenstra Toussaint. Natuurkundig tijdschrift voor Nederlandsch-Indië, 16, $42-59$.

Blum, J.P., Menzies, J.I. (1989) Notes on Xenobatrachus and Xenorhina (Amphibia: Microhylidae) from New Guinea with description of nine new species. Alytes, 7, 125-163.

Boie, F. (1827) Bemerkungen über Merrem's Versuch eines Systems der Amphibien. 1te Lieferung: Ophidier. Isis von Oken, 1827, 508-566.

Bokermann, W.C.A. (1966a) Lista anotada das localidades tipo de anfíbios brasileiros. São Paulo, 183 pp.

Bokermann, W.C.A. (1966b) Two new species of Physalaemus from Espirito Santo, Brasil (Amphibia, Leptodactylidae). Physis (Buenos Aires), 26, 193-202.

Bokermann, W.C.A. (1966c) Una nueva especie de Trachycephalus de Bahia, Brasil (Amphibia, Hylidae). Neotropica, $12,120-124$.

Bossuyt, F., Dubois, A. (2001) A review of the frog genus Philautus Gistel, 1848 (Amphibia, Anura, Ranidae, Rhacophorinae). Zeylanica, 6, 12.

Boulenger, G.A. (1882) Catalogue of the Batrachia Salientia s. Ecaudata in the Collection of the British Museum (2nd ed.). London, $503 \mathrm{pp}$.

Boulenger, G.A. (1887) Description of new or little-known South American frogs of the genera Paludicola and Hyla. Annals and Magazine of Natural History, Ser. 5, 296.

Boulenger, G.A. (1896) Description of new reptiles and batrachians from Colombia. Annals and Magazine of Natural History, Ser. 6, 17, 16-21.

Brongersma, L.D. (1948) Frogs and snakes from the island of Morotai (Moluccas). Zoologische Mededelingen Leiden, 29, 306-3 10.

Brongersma, L.D. (1953) Notes on New Guinea reptiles and amphibians III. Proceedings of the Koninklijke Nederlandse Akademie van Wetenschappen, Serie C, 56, 572-582.

Brown, W.C., Parker, F. (1970) New frogs of the genus Batrachylodes (Ranidae) from the Solomon Islands. Breviora, $346,1-31$.

Cannatella, D.C. (1984) Two new species of the leptodactylid frog genus Phrynopus, with comments on the phylogeny of the genus. Occasional Papers of the Museum of Natural History University of Kansas, 113, 1-16.

Capocaccia, L. (1957) Catalogo dei tipi di anfibi del Museo Civico di Storia Naturale di Genova. Annali del Museo Civico di Storia Naturale di Genova, 208-222.

Cei, J.M. (1962) Batracios de Chile. Universidad de Chile, Santiago de Chile, 128 pp.

Cogger, H.G., Cameron, E.E., Cogger, H.M. (1983) Zoological Catalogue of Australia. Vol. 1. Canberra, 313 pp.

Coloma, L.A. (1995) Ecuadorian frogs of the genus Colostethus (Anura: Dendrobatidae). Miscellaneous Publications of the Museum of Natural History University of Kansas, 87, 1-72.

Dubois, A., Ohler, A. (1998) A new species of Leptobrachium (Vibrissaphora) from northern Vietnam, with a review of the taxonomy of the genus Leptobrachium (Pelobatidae, Megophryinae). Dumerilia, 4, 1-32.

Dubois, A., Ohler, A. (2000) Systematics of Fejervarya limnocharis (Gravenhorst, 1829) (Amphibia, Anura, Ranidae) and related species. 1. Nomenclatural status and type-specimens of the nominal species Rana limnocharis Gravenhorst, 1829. Alytes, 18, 15-50.

Duellman, W.E. (1972) South American frogs of the Hyla rostrata group (Amphibia, Anura, Hylidae). Zoologische Mededelingen Leiden, 47, 177-192.

Duellman, W.E. (1977) Liste der rezenten Amphibien und Reptilien: Hylidae, Centrolenidae, Pseudidae. Walter de Gruyter, Berlin, 225 pp.

Duellman, W.E. (1986) Two new species of Ololygon (Anura: Hylidae) from the Venezuelan Guyana. Copeia, 864-870.

Duellman, W.E., Hoogmoed, M.S. (1984) The taxonomy and phylogenetic relationships of the hylid frog genus Stefania. Miscellaneous Publications of the Museum of Natural History University of Kansas, 75, 1-35.

Duellman, W.E., Simmons, J.E. (1977) A new species of Eleutherodactylus (Anura: Leptodactylidae) from the Cordillera Oriental of Colombia. Proceedings of the Biological Society of Washington, 90, 60-65.

Duellman, W.E., Wiens, J.J. (1993) Hylid frogs of the genus Scinax Wagler, 1830, in Amazonian Ecuador and Peru. Occasional Papers of the Museum of Natural History University of Kansas, 153, 1-57.

Duméril, A.M.C., Bibron, G. (1841) Erpétologie générale ou histoire naturelle complète des reptiles. Tome 8. Roret, Paris, 792 pp. 
Dunn, E.R. (1926) Additional frogs from Cuba (Contrib. Smith. Collage Dept. Zool. 139). Occasional Papers of the Boston Society of Natural History, 5, 209-215.

Fitzinger, L.I. (1826) Neue Classification der Reptilien nach iheren naturlichen Verwandtschaften. Nebst einer Verwandtschaftstafel uhnd einem Verzeichnisse der Reptilien der Reptilien-Sammlung des K.K. Zoologischen Museums in Wien. Vienna, $66 \mathrm{pp}$.

Frost, D.R. (1985) Amphibian species of the world: a taxonomic and geographical reference. Allen Press, Lawrence, KS, $732 \mathrm{pp}$.

Frost, D.R. (2006) Amphibian Species of the World: an Online Reference. Version 4 (17 August 2006). Electronic Database accessible at http://research.amnh.org/herpetology/amphibia/index.php. American Museum of Natural History, New York, USA.

Gallardo, J.M. (1965) The species Bufo granulosus Spix (Salienta: Bufonidae) and its geographic variation. Bulletin of the Museum of Comparative Zoology, 134, 107-138.

Goin, C.J. (1966a) Description of a new frog of the genus Hyla from Suriname. Zoologische Mededelingen Leiden, 41, 229-232.

Goin, C.J. (1966b) A new frog of the genus Centrolenella from Suriname. Studies on the fauna of Suriname and other Guyanas, 8, 77-80.

Gravenhorst, J.L.C. (1829) Deliciae Musei Zoologici Vratislaviensis (Reptilia Musei Zoologici Vratislaviensis. Recensita et Descripta). Fasciculus Primus, continens Chelonions et Batrachia: I-XIV. Leopold Voss, Leipzig, 106 pp.

Guibé, J. (1950) Catalogue des types d'Amphibiens du Muséum National d'Histoire Naturelle. Imprimerie nationale, Paris, $71 \mathrm{pp}$.

Häupl, M., Tiedemann, F., Grillistsch, H. (1994) Katalog der Typen der Herpetologischen Sammlung nach dem Stand vom 1. Selbstverlag Naturhistoriches Wien, Vienna, $46 \mathrm{pp}$.

Heyer, W.R. (1973) Systematics of the marmoratus group of the frog genus Leptodactylus (Amphibia, Leptodactylidae). Contributions in Science, Natural History Museum of Los Angeles County, 251, 1-50.

Heyer, W.R. (2005) Variation and taxonomic clarification of the large species of the Leptodactylus pentadactylus species group (Amphibia: Leptodactylidae) from Middle America, Northern South America and Amazonia. Arquivos de Zoologia, 37, 269-348.

Holthuis, L.B., Sakai, T. (1970) Ph. F. von Siebold and Fauna japonica: a history of early Japanese zoology. Academic Press of Japan ,Tokyo, $323 \mathrm{pp}$.

Hoogmoed, M.S. (1969) Notes on the herpetofauna of Surinam III. A new species of Dendrobates (Amphibia, Salientia, Dendrobatidae) from Surinam. Zoologische Mededelingen Leiden, 44, 133-141.

Hoogmoed, M.S. (1972) On a new species of toad from Southern Morocco. Zoologische Mededelingen Leiden, 47, 4964.

Hoogmoed, M.S. (1978) An annotated review of the salamander types described in the Fauna Japonica. Zoologische Mededelingen Leiden, 53, 91-105.

Hoogmoed, M.S. (1985) A new genus of toads (Amphibia: Anura: Bufonidae) from the Pacific slopes of the Andes in Northern Ecuador and Southern Colombia, with the description of two new species. Zoologische Mededelingen Leiden, 59, 251-274.

Hoogmoed, M.S. (1986) Additional remarks on Spix types in the Rijksmuseum van Natuurlijke Historie, Leiden. Zoologische Mededelingen Leiden, 60, 299-300.

Hoogmoed, M.S. (1990) Rediscovery of a type of Ceratophrys boiei Wied (Amphibia: Anura: Leptodactylidae). Zoologische Mededelingen Leiden, 63, 267-273.

Hoogmoed, M.S., Borges, D.M., Cascon, P. (1994) Three new species of the genus Adelophryne (Amphibia: Anura: Leptodactylidae) from Northeastern Brazil, with remarks on the other species of the genus. Zoologische Mededelingen Leiden, 68, 271-300.

Hoogmoed, M.S., Gorzula, S.J. (1979) Checklist of the savanna inhabiting frogs of the El Manteco Region with notes on their ecology and the description of a new species of treefrog (Hylidae, Anura). Zoologische Mededelingen Leiden, 54, 183-216.

Hoogmoed, M.S., Gruber, U. (1983) Spix and Wagler type specimens of reptiles and amphibians in the Natural History Musea in Munich (Germany) and Leiden (The Netherlands). Spixiana, 9, 319-415.

Hoogmoed, M.S., Lescure, J. (1984) A new genus and two new species of minute leptodactylid frogs from Northern South America, with comments upon Phyzelaphryne (Amphibia: Anura: Leptoldactylidae). Zoologische Mededelingen Leiden, 58, 85-115.

Horst, R. (1883) On new and little-known frogs from the Malayan Archipelago. Notes of the Leyden Museum, 5, 235244.

ICZN (1994) Opinion 1763. Megophrys montana Kuhl \& Van Hasselt, 1822 (Amphibia, Anura): generic and specific names placed on Official Lists, and Leptobrachium parvum Boulenger, 1893 (currently Megophrys parva): specific name conserved. Bulletin of Zoological Nomenclature, 51, 84-85.

Inger, R.F. (1960) A review of the Oriental toads of the genus Ansonia Stoliczka. Fieldiana, Zoology, 39, $473-503$. 
Inger, R.F. (1964) Two new species of frogs from Borneo. Fieldiana, Zoology, 44, 151-159.

Inger, R.F. (1966) The systematics and zoogeography of the Amphibia of Borneo. Fieldiana, Zoology, 52, 1-402.

Inger, R.F., Stuebing, R.B. (1996) Two new species of frogs from Southeastern Sarawak. The Raffles Bulletin of Zoology, 44, 543-549.

Kuhl, H., Van Hasselt, J.C. (1822a) Aus einem Schreiben von Dr. Kuhl und Dr. Van Hasselt aus Java. Isis von Oken, 4, 472-476.

Kuhl, H., Van Hasselt, J.C. (1822b) Uittreksels uit brieven van de Heeren Kuhl en Van Hasselt, aan de Heeren C.J. Temminck, Th. van Swinderen en W. de Haan. Algemeene Konst- en Letterbode, 7, 99-104.

Laurenti, J.N. (1768) Specimen medicum, exhibens synopsin Reptilium emendatum cum experimentis circa venana et antidota reptilium Austriacorum. Joan, Thom. Nob. de Trattnern, Vienna, 214 pp., V plates.

Lescure, J. (1976) Contrubution à l'étude des Amphibiens de Guyane française. VI. Liste préliminaire des Anoures. Bulletin du Muséum National d'Histoire Naturelle, (3) 377, 475-524.

Lescure, J. (1981) Contribution à l'étude des Amphibiens de Guyane française. VIII. Validation d'Atelopus spumarius Cope, 1871, et désignation d'un néotype. Description d'Atelopus spumarius barbotini nov. ssp. Données éthoécologiques et biogéographiques sur les Atelopus dus groupe flavescens (Anoures, Bufonidés). Bulletin du Muséum National d'Histoire Naturelle, Ser. 4(A), 3, 893-910.

Lescure, J. (1988) Les types d'amphibiens gymnophiones du Museum National d'Histoire naturelle: catalogue critique. Bulletin du Muséum National d'Histoire Naturelle, Sect. A, 10, 59-71.

Lesson, R.P. (1827) Voyage autour du monde, exécuté par ordre du roi, sur la corvette de Sa Majesté, La Coquille, pendant les années 1822, 1823, 1824 et 1825: sous le Ministère et conformément aux instructions de S. E. M. le Marquis Clermont-Tonnerre, ministre de la marine, sous la dir. de L.I. Duperrey et Garnot. Zoologie du voyage autour du monde, exécuté sur la corvette du Roi la Coquille: tome second. Arthus Bertrand, Paris, 471 pp.

Loveridge, A.L. (1935) Scientific results of an expedition to rain forest regions in Eastern Africa. I. New reptiles and amphibians from East Africa. Bulletin of the Museum of Comparative Zoology, 79, 1-19.

Loveridge, A.L. (1953) Zoological results of a Fifth Expedition to East Africa. IV. Amphibians from Nyasaland and Tete. Bulletin of the Museum of Comparative Zoology, 110, 323-406.

Lynch, J.D., Hoogmoed, M.S. (1977) Two new species of Eleutherodactylus (Amphibia: Leptodactylidae) from Northeastern South America. Proceedings of the Biological Society of Washington, 90, 424-439.

Lynn, W.G. (1937) Two new frogs from Jamaica. Herpetologica, 1, 89-91.

Matsui, M. (1984) Morphometric variation analyses and revision of the Japanese toads (genus Bufo, Bufonidae). Contributions from the Biological Laboratory Kyoto University, 26, 209-428.

Mertens, R. (1930) Die von Dr. F. Kopstein auf den Molukken und einigen benachbarten Inseln gesammelten Froschlurche. Zoologische Mededelingen Leiden, 13, 141-150.

Michahelles, C.W. (1830) Neue südeuropäische Amphibien. Isis von Oken, 23, 189-195.

Müller, L. (1927) Amphibien und Reptilien der Ausbeute Prof. Bresslau's in Brasilien 1913-1914. Abhandlungen der Senckenbergischen Naturforschenden Gesellschaft, 40, 259-304.

Müller, S. (1833) Aanteekeningen gehouden op eene reize over een gedeelte van het eiland Java, door de leden der Natuurkundige Kommissie (wijlen) P. van Oort \& S. Müller, in den aanvang van het jaar 1833. Verhandelingen van het Bataviaasch Genootschap van Kunsten en Wetenschappen, 16, 81-156.

Nussbaum, R.A., Hoogmoed, M.S. (1979) Surinam Caecilians, with notes on Rhinatrema bivittatum and the description of a new species of Microcaecilia (Amphibia, Gymnophiona). Zoologische Mededelingen Leiden, 54, 218-235.

Ortiz, J.C., Lescure, J. (1989) Les types d'amphibiens anoures du Chili dans les collections du Museum National d'Histoire naturelle. Catalogue critique. Bulletin du Muséum National d'Histoire Naturelle, 3, 113-122.

Parker, H.W. (1934) A monograph on the frogs of the family Microhylidae. British Museum (Natural History), London, 208 pp.

Parker, H.W. (1936) Amphibians from Liberia and the Gold Coast. Zoologische Mededelingen Leiden, 19, 87-102.

Parker, H.W. (1940) The Australian frogs of the family Leptodactylidae. Novitates Zoologicae, 42, 1-106.

Perret, J.L. (1994) Revision of the genus Aubria Boulenger 1917 (Amphibia Ranidae) with the description of a new species. Tropical Zoology, 7, 255-269.

Peters, W.C.H. (1854) Diagnosen neuer Batrachier, welche zusammen met dir früher (24 Juli und 17 August) gegebenen Übersicht der Schlangen und Eidechsen mitgetheilt werden. Bericht über die zur Bekanntmachung geeigneten Verhandlungen der Königlich Preußischen Akademie der Wissenschaften zu Berlin, 614-628.

Peters, W.C.H. (1867) Herpetologische Notizen. Monatsberichte der Königlich Preußischen Akademie der Wissenschaften zu Berlin, 13-37.

Peters, W.C.H., Doria, G. (1878) Catalogo dei rettili e dei batraci raccolti da O. Beccari, L.M. D'Albertis e A.A. Bruijn nella Sotto-Regione Austro-Malese. Annali del Museo Civico di Storia Naturale di Genova, 13, 323-450.

Poynton, J.C. (1963) Description of southern African amphibians. Annals of the Natal Museum, 14, 319-332. 
Roux-Estève, R. (1979) Liste des amphibiens et reptiles des collections du Muséum National d'Histoire Naturelle de Paris, récóltes par Lesueur (1778-1846). Bulletin Trimestriel de la Société Géologique de Normandie et des Amis du Muséum du Havre, 66, 25-29.

Schlegel, H. (1837-1844) Abbildungen neuer oder unvollständig bekannter Amphibien, nach der Natur oder dem Leben entworfen. Arnz \& Comp., Düsseldorf, 141 pp. Atlas, 49 plates.

Schlegel, H. (1841) Bruchstücke in einer Fauna der Berberei. In: Wagner (Ed.) Reisen Regentschaft Algier, $3,134$.

Schlegel, H. (1850) Description of a new genus of Batrachians from Swan River. Proceedings of the Zoological Society of London, 18, 9-10.

Schlegel, H. (1858) Handleiding tot de beoefening der dierkunde. Breda, $628 \mathrm{pp}$.

Smith, M.A. (1927) Contributions to the herpetology of the Indo-Australian Region. Proceedings of the Zoological Society of London, 1927, 199-225.

Spix, J.B.v. (1824) Animalia nova sive species novae Testudinum et Ranarum, quas in itinere per Brasiliam annis MDCCCXVII-MDCCCXX. Hübschmanni, Munich, 53 pp., XXII plates.

Stejneger, L. (1907) Herpetology of Japan and adjacent territory. Bulletin of the United States National Museum, 58, 1577.

Stresemann, E. (1951) Die Entwicklung der Ornithologie von Aristoteles bis zur Gegenwart. English translation by Epstein, 1975. Ornithology from Aristotle to the present. Ed. by G.W. Cottrell. Harvard University Press, Cambridge, Massachusetts, $432 \mathrm{pp}$.

Taylor, E.H. (1965) New Asiatic and African caecilians with redescription of certain other species. The Kansas University Science Bulletin, 46, 253-302.

Taylor, E.H. (1968) The caecilians of the world: a taxonomic review. University of Kansas Press, Lawrence, 848 pp.

Temminck, C.J. (1836) Coup-d'oeil sur la faune des iles de la Sonde et de l'empire du Japon. Discours preliminaire destine a servir d'introduction a la Faune du Japon. In: Siebold, P.F.v. (Ed.) Fauna Japonica sive Descriptio animalium, quae in itinere per Japoniam, jussu et auspiciis Superiorum, qui summum in India Batava imperium tenent, suscepto annis 1823-1830, collegit, notis, observationibus et adumbrationibus illustravit, Lugduni Batavorum, pp. i-xxx.

Temminck, C.J., Schlegel, H. (1838) Fauna Japonica. Vol. 3. In: Siebold, P.F.v. (Ed.) Fauna Japonica sive Descriptio animalium, quae in itinere per Japoniam, jussu et auspiciis Superiorum, qui summum in India Batava imperium tenent, suscepto annis 1823-1830, collegit, notis, observationibus et adumbrationibus illustravit, Lugduni Batavorum, $329 \mathrm{pp}$.

Tschudi, J.J.v. (1837) Über den Homo diluvii testis, Andrias Scheuchzeri. Neues Jahrbuch für Mineralogie, Geognosie, Geologie und Petrefaktenkunde, 5: 545-547.

Tschudi, J.J.v. (1838) Classification der Batrachier, mit Berücksichtigung der fossilen Thiere. Neuchatel, 99 pp., plates IVI.

Tyler, M.J. (1968) Papuan hylid frogs of the genus Hyla. Zoologische Verhandelingen Leiden, 96, 1-202.

Van der Laan, J., ed. (2001). The history of natural history in Leiden. National Museum of Natural History, Leiden, 78 pp.

Van Kampen, P.N. (1906) Résultats de l'expédition scientifique Néerlandaise à la Nouvelle-Guinée en 1903.17 Amphibien. Nova Guinea, 5, 163-180.

Van Kampen, P.N. (1923) Amphibia of the Indo-Australian Archipelago. E.J. Brill Ltd., Leiden, 304 pp.

Van Lidth de Jeude, T.W. (1904) Reptiles and Batrachians from Surinam. Notes of the Leyden Museum, $25,83-94$.

Van Tuijl, L. (1995) Revised catalogue of the type specimens of recent amphibians and reptiles in the "Zoölogisch Museum" University of Amsterdam, The Netherlands. Bulletin Zoölogisch Museum Universiteit van Amsterdam 14, $125-141$.

Wagler, J.G. (1824) Serpentum Brasiliensium species novae ou Histoire Naturelle des espèces nouvelles de serpens, recueillies et observées pendant le voyage dans l'intérieur de Brésil dans les années 1817, 1818, 1819, 1820 exécuté par Sa Majesté le Roi de Bavière / publiée par Jean de Spix; écrite d'après les notes du voyageur par Jean Wagler. Hübschmann, Munich, 75 pp., XXVI plates.

Wagler, J.G. (1827) H. Boie an Wagler. Buitenzorg aus Java ben A25 Aug. 1825. Isis von Oken, 20, 724-726.

Wied-Neuwied, M.P.v. (1824) Berzeinhniß der Amphibien, welche im zwenten Bande der Naturgeschichte Brasiliens von Prinz Max von Neuwied werden beschrieben werden. Isis von Oken, 14, 661-673.

Wied-Neuwied, M.P.v. (1825) Beiträge zur Naturgeschichte von Brasilien I. Weimar, 612 pp.

Wiegmann, A.F.A. (1834) Beiträge zur Zoologie, gesammelt auf einer Reise um die Erde, von Dr. F. J. F. Meyen, M.d.A.d.N. Amphibien.Amphibien. Nova Acta Physico-medica Academiae Caesareae Leopoldino-Carolinae Naturae Curiosorum, 17 (1): 183-268, plates XIII-XXII.

Zweifel, R.G. (1956) Results of the Archbold Expeditions No. 72. Microhylid frogs from New Guinea, with descriptions of new species. American Museum Novitates, 1766, 1-49.

Zweifel, R.G. (1967) Identity of the frog Cornufer unicolor and applicatioin of the generic name Cornufer. Copeia, 1967, 117-121. 
Zweifel, R.G. (1972a) Results of the Archbold Expeditions No. 97. A revision of the frogs of the subfamily Asterophryinae family Microhylidae. Bulletin of the American Museum of Natural History, 148, 411-546.

Zweifel, R.G. (1972b) A review of the frog genus Lechriodus (Leptodactylidae) of New Guinea and Australia. American Museum Novitates, 2507, 1-41. 